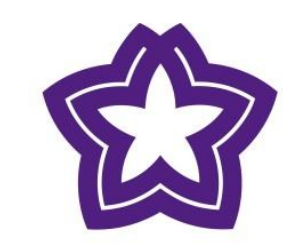

DALARNA
UNIVERSITY

Working papers in transport, tourism, information technology and microdata analysis

\title{
On statistical bounds of heuristic solutions to location problems
}

Författare 1: Kenneth Carling Författare 2: Xiangli Meng

Nr: 2014:10

Editor: Hasan Fleyeh

(C) Authors 


\title{
On statistical bounds of heuristic solutions to location problems
}

\author{
Authors : Kenneth Carling and Xiangli Meng*
}

\begin{abstract}
Solutions to combinatorial optimization problems, such as problems of locating facilities, frequently rely on heuristics to minimize the objective function. The optimum is sought iteratively and a criterion is needed to decide when the procedure (almost) attains it. Pre-setting the number of iterations dominates in OR applications, which implies that the quality of the solution cannot be ascertained. A small, almost dormant, branch of the literature suggests using statistical principles to estimate the minimum and its bounds as a tool to decide upon stopping and evaluating the quality of the solution. In this paper we examine the functioning of statistical bounds obtained from four different estimators by using simulated annealing on $p$-median test problems taken from Beasley's OR-library. We find the Weibull estimator and the $2^{\text {nd }}$ order Jackknife estimator preferable and the requirement of sample size to be about 10 being much less than the current recommendation. However, reliable statistical bounds are found to depend critically on a sample of heuristic solutions of high quality and we give a simple statistic useful for checking the quality. We end the paper with an illustration on using statistical bounds in a problem of locating some 70 distribution centers of the Swedish Post in one Swedish region.
\end{abstract}

Key words: $p$-median problem, simulated annealing, jack-knife, discrete optimization, extreme value theory

\footnotetext{
- Kenneth Carling is a professor in Statistics and Xiangli Meng is a PhD-student in Micro-data analysis at the School of Technology and Business Studies, Dalarna university, SE-791 88 Falun, Sweden.

* Corresponding author. E-mail: xme@du.se. Phone: +46-23-778509. 


\section{Introduction}

Consider the problem of finding a solution to $\min _{\Theta} f(\Theta)$ where the complexity of the function renders analytical solutions infeasible. To focus ideas, consider the $p$-median problem on the network. The problem is to allocate $P$ facilities to a population geographically distributed in $Q$ demand points such that the population's average or total distance to its nearest service center is minimized. Hakimi (1964) considered the task of locating telephone switching centers and showed later (Hakimi, 1965) that, in a network, the optimal solution of the $p$-median model existed at the nodes of the network. If $N$ is the number of nodes, then there are $\left(\begin{array}{l}N \\ P\end{array}\right)$ possible solutions for a p-median problem.

As enumerating all possible solutions is not possible as the problem size grows, much research has been devoted to efficient (heuristic) algorithms to solve the $p$-median model (see Handler and Mirchandani, 1979 and Daskin, 1995 as examples). In this work we will concentrate on a common heuristic known as simulated annealing and we follow the implementation of Lenanova and Loresh (2004). ${ }^{1}$ The virtue of simulated annealing as other heuristics is that the algorithm will iterate towards a good solution, not necessarily the actual optimum.

The prevailing practice is to run the heuristic algorithm for a pre-specified number of iterations or until improvements in the solution becomes infrequent. Such practice does not readily lend itself to determine the quality of the solution in a specific problem. One strategy to assess the quality is to seek deterministic bounds for the minimum by techniques such as Lagrangian Relaxation (see Beasley, 1993). This strategy is popular and reasonable for many problems, but the deterministic bounds depend on the chosen parameters and are available for only a limited set of heuristic algorithms.

An alternative strategy to deterministic bounds is statistical bounds. In short, the statistical approach is to estimate the value of the minimum based on a sample of heuristic solutions and put confidence limits around it. Golden and Alt (1979) did pioneering work on statistical bounds followed by others in the 1980:s, but thereafter the statistical approach has received little interest. In fact, Akyüz, Öncan,

\footnotetext{
${ }^{1}$ Simulated annealing was implemented in $R$ (www.r-project.org) and the code is attached in the Appendix. 
and, Altınel (2012) state that the statistical approach had not been used in location problems since 1993 to the best of their knowledge.

However, the usefulness of statistical bounds, as discussed by for instance Derigs (1985), presumably still applies and they therefore merit a critical examination. There are a number of open questions regarding statistical bounds and their implementation. How to estimate the minimum? How and according to which principle should the bounds be derived? What is the required sample size? Are they reliable? Are they computationally affordable? Does the choice of heuristic matter? How do they perform in various OR-problems? To address all these questions at once would be an insurmountable task, and therefore we limit the analysis to the first four questions in connection to the $p$-median problem solved by simulated annealing. A positive answer to these four questions is a prerequisite for the latter three questions to be worthwhile to address. Specifically, the aim of this paper is to test by experimentation if statistical bounds can provide information on the optimum in $p$-median problems solved by simulated annealing.

This paper is organized as follows: in section two we review suggested methods for statistically estimating the minimum of the objective function as well as bounds for the minimum and add some new remarks on the issue. In the third section we compare the methods by applying them to uncapacitated $p$-median test problems of a known optimum of varying complexity and such test problems are available from the Beasley OR-library (Beasley, 1990). Section four presents a real-world problem of locating distribution centres for the Swedish Post in a region in mid-Sweden. The fifth section concludes this paper.

\section{Statistical estimation of the minimum and its bounds}

From a statistical point of view solving a $p$-median problem means identifying the smallest value of a distribution. First some notations used throughout the paper:

$z_{p}=$ feasible solution of locating $P$ facilities in $N$ nodes.

$A=$ the set of all feasible solutions $A=\left\{z_{1}, z_{2}, \ldots, z_{\left(\begin{array}{l}N \\ P\end{array}\right)}\right\}$.

$g\left(z_{p}\right)=$ the objective function of solution $z_{p}$. 
$\theta=\min _{A} g\left(z_{p}\right)$

The challenge is to identify $\theta$ and the corresponding solution $z_{p}$. Because of the complexity of the problem most of the times one can only hope to get a solution near to $\theta$. Statistical bounds would add information about the solution by, ideally, providing an interval that almost certainly contains $\theta$. We will consider the extreme value theory (EVT) approach in which the distribution of the solution is assumed to be the Weibull distribution (Derigs, 1985). We will complement the EVT approach by also considering the possibility that the solution's distribution is Gumbel. Finally, to loosen the distributional assumptions of the EVT approaches we will also consider the non-parametric methods Jackknifing and bootstrapping. The computational cost of calculating statistical bounds by all the approaches is trivial compared to solving the $p$-median problem and we will ignore this cost in the following.

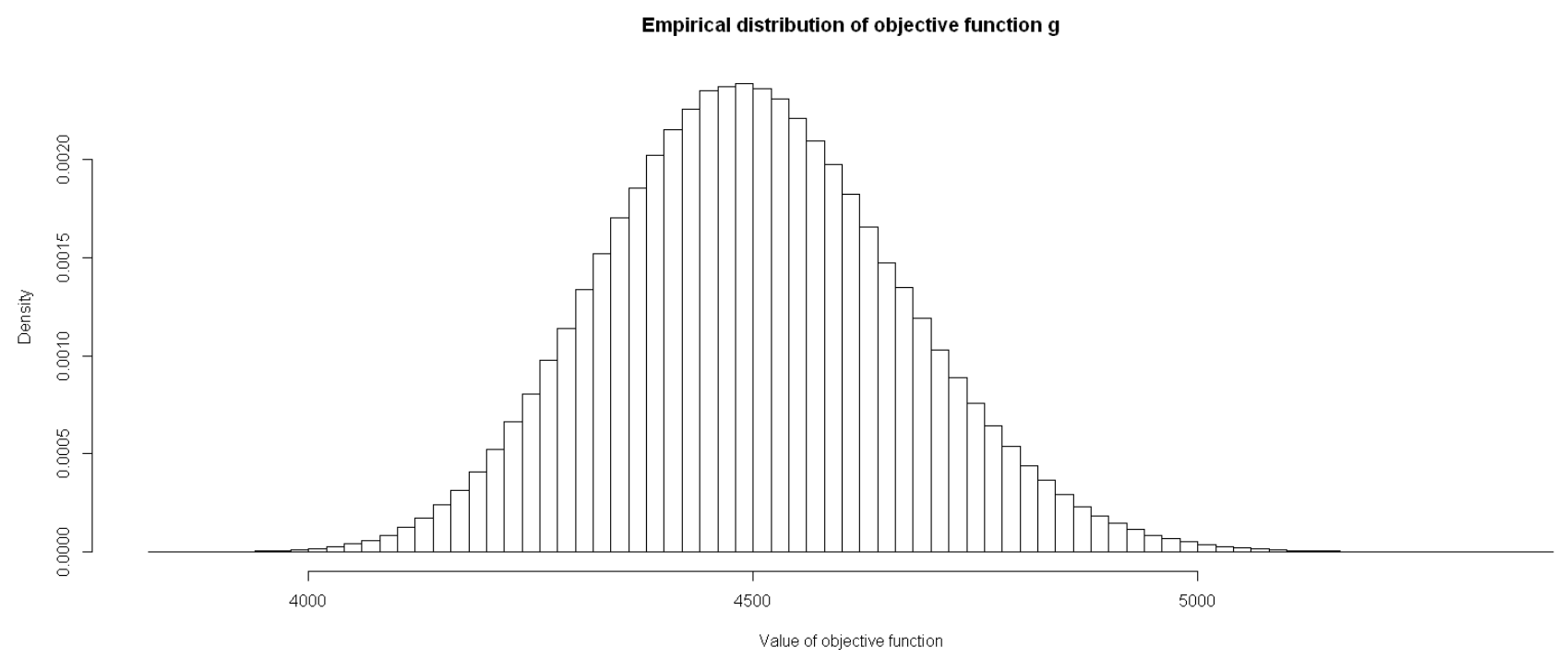

Figure 1: Sample distribution of the $14^{\text {th }}$ problem in the OR-library.

Figure 1 gives an example of the distribution of feasible solutions to a $p$-median problem being the $14^{\text {th }}$ problem in the OR-library (Beasley, 1990). One million $z_{p}$ 's are drawn at random from $A$ and the histogram of the corresponding $g\left(z_{p}\right)$ is given. This empirical distribution as well as the distributions of most of the other problems in the OR-library mimics the Normal distribution. However, this sample is almost useless for identifying $\theta$. The crux is the required size of the subset of $A$. The objective function in $p$-median problems might be regarded as approximately Normal with a truncation in the left tail being the minimum $\theta$. For a good estimate of $\theta$ feasible solutions near - 5 - 
to $\theta$ would be required. For $\theta$ far out in the tail, the subset of $A$ would need to be huge. We show below that for many of the OR-library $p$-median problems, the minimum is at least some 6 standard deviations away from the mean requiring a subset of a size of $1 / \Phi(-6) \approx 10^{9}(\Phi$ is the standard Normal distribution function) to render hope of having feasible solutions close to $\theta$.

Fortunately, as several authors have pointed out, if the starting values are picked at random, repeated heuristic solutions mimic a random sample in the tail (see e.g. McRoberts, 1971 and Golden and Alt, 1979). Thereby random values in the tail can be obtained at a much less computational effort and used for estimating $\theta$. To discuss estimation of $\theta$ and statistical bounds for the parameter we need additional notations:

$\hat{\theta}=$ an estimator of $\theta$.

$n=$ the number of runs of the heuristic algorithm with random starting values.

$\tilde{x}_{i}=$ the heuristic solution of $i^{\text {th }}$ run $i=1,2, \ldots, n$.

The Weibull and the Gumbel estimators are $\hat{\theta}_{W}=\hat{\theta}_{G}=\tilde{x}_{(1)}$ where $\tilde{x}_{(1)}$ is the best (smallest) heuristic solution in all the $n$ runs. An alternative, briefly discussed in the literature, is the Jackknifing approach (hereafter JK). The JK-estimator is introduced by Quenouille (1956):

$$
\hat{\theta}_{J K}=\sum_{i=1}^{M+1}(-1)^{(i-1)}\left(\begin{array}{c}
M+1 \\
i
\end{array}\right) \tilde{x}_{(i)}
$$

where $M$ is the order. Dannenbring (1977) and Nydick and Weiss (1988) suggest to use the first order, i.e. $M=1$, for point estimating the minimum. The first order JK-estimator is more biased than higher order ones, but its mean square error is lower compared with higher orders as shown by Robson and Whitlock (1964). We will however consider both the first and the second order JK-estimator. The point-estimators are $\hat{\theta}_{J K}^{(1)}=2 \tilde{x}_{(1)}-\tilde{x}_{(2)}$ and $\hat{\theta}_{J K}^{(2)}=3 \tilde{x}_{(1)}-3 \tilde{x}_{(2)}+\tilde{x}_{(3)}$ respectively.

The upper bounds of the Weibull, the Gumbel, and the Jackknifing approaches are the same, $\tilde{x}_{(1)}$. However their lower bounds differ. The Weibull lower bound with a confidence of $100\left(1-e^{-n}\right) \%$ is $\left[\tilde{x}_{(1)}-\hat{b}\right]$ where $\hat{b}$ is the estimated shape parameter of the Weibull distribution (Wilson, King and Wilson, 2004). There are several ways of estimating the shape parameter, including the $-6-$ 
maximum likelihood estimation technique. We found the following simple estimator to be fast, stable, and giving good results: $\hat{b}=\tilde{x}_{[0.63(n+1)]}-\left(\tilde{x}_{(1)} \tilde{x}_{(n)}-\tilde{x}_{(2)}{ }^{2}\right) /\left(\tilde{x}_{(1)}+\tilde{x}_{(n)}-2 \tilde{x}_{(2)}\right)$ where $[0.63(n+1)]$ means the integer value of the function (Derigs, 1985). The justification for the Weibull approach is a belief that $g\left(z_{p}\right)$ follows a skewed (or a uniform distribution). However as mentioned above, we took large random subsets of $A$ for the 40 problems in the OR-library and found that $g\left(z_{p}\right)$ is typically symmetrically distributed and only slightly skewed in instances in which $P$ is small. As a consequence of this proximity to the Normal distribution, the extreme values might better be modelled by the Gumbel distribution (see Kotz and Nadarajah, 2000 (p. 59)).

We derive the Gumbel lower bound by its theoretic percentile $[\mu-\sigma \ln (-\ln (1-\alpha))]$ where $\mu$ and $\sigma$ are the location and shape parameters of the Gumbel distribution while $\alpha$ is the confidence level. We estimate the Gumbel-parameters by the moments as $\widehat{\sigma}=\frac{\sqrt{6 \operatorname{var}\left(\tilde{x}_{i}\right)}}{\pi}$ and $\hat{\mu}=\overline{\tilde{x}}_{l}-$ 0.57722 $\widehat{\sigma}$ where details are in Kotz and Nadarajah (2000, p. 12).

In the Weibull approach the confidence level is determined by $n$. To render the Weibull and the Gumbel approach comparable in terms of confidence level we will let $\alpha=e^{-n}$.

To produce a Jackknifing lower bound we suggest the bootstrap method by Efron (1979). The lower bound is $\left[\hat{\theta}_{J K}-3 \sigma^{*}\left(\hat{\theta}_{J K}\right)\right]$ where $\sigma^{*}\left(\hat{\theta}_{J K}\right)$ is the standard deviation of $\hat{\theta}_{J K}$ obtained from bootstrapping the $n$ heuristic solutions (1,000 times we found sufficient). With the scalar 3, the confidence is $99.9 \%$ provided that the sampling distribution of the JK-estimator is Normal.

\section{Experimental evaluation of the estimators}

The two EVT-estimators Weibull and Gumbel $\left(\hat{\theta}_{W}, \hat{\theta}_{G}\right)$, and the JK-estimators $\hat{\theta}_{J K}$ and the accompanying bounds are justified by different arguments and there is no way to deem one superior to the others unless they are put to test. We have not found any previous work comparing the estimators so we use the OR-library problems for an experimental comparison of them since these 40 test problems enables a comparison with respect to their known minimum $\theta$. Moreover, the 
problems vary substantially in (statistical) complexity, define here by us as $\left(\left(\mu_{g\left(z_{p}\right)}-\theta\right) / \sigma_{g\left(z_{p}\right)}\right)$, as their minimum are varyingly far out in the tail of the distribution of $g\left(z_{p}\right)$. We focus on the estimators' ability to generate intervals that covers the problems' minimum as well as the length of the intervals. Most details in calculating the estimators and the bounds were given in section 2, except for the size of $n$ and the required number of iterations of the heuristic algorithm. Based on pre-testing the heuristic algorithm, we decided to evaluate the estimators after 1,000, 10,000, and, for the more complex problems, 100,000 iterations. In the early literature on statistical bounds little was said on the size of $n$, whereas Akyüz et al (2012) and Luis, Sahli, and Nagy (2009) advocate $n$ to be at least 100 . We shall examine $n=3,10,100$ and if deemed warranted based on the experimental results we will consider even larger values of $n$.

\subsection{The complexity of the test problems}

In Table 1 we give $\theta, \mu_{g\left(z_{p}\right)}, \sigma_{g\left(z_{p}\right)}$ and the complexity of the problems where the parameters for the mean and the standard deviation are estimated on a random subset of 1,000,000 from $A$. Instead of following the original order of the test problems, Table 1 gives the problems in ascending order of complexity (see also Table A1). The complexity varies between 2.56 for problem P11 to 14.93 for problem P30.

Table 1: Description of 6 problems from the OR-library.

\begin{tabular}{|lcccc|}
\hline \hline Problem & $\theta$ & $\mu_{g\left(z_{p}\right)}$ & $\sigma_{g\left(z_{p}\right)}$ & Complexity \\
\hline P11 & 7696 & 10760 & 1195 & 2.56 \\
P2 & 4093 & 6054 & 506 & 3.88 \\
P36 & 9934 & 13436 & 735 & 4.77 \\
P13 & 4374 & 6293 & 276 & 6.95 \\
P33 & 4700 & 6711 & 186 & 10.81 \\
P30 & 1989 & 3335 & 90 & 14.93 \\
\hline
\end{tabular}

All estimators require an efficient heuristic that produces random solutions in the very tail of the distribution $g\left(z_{p}\right)$. In the experiments we consistently generate random solutions, $\tilde{x}_{i}$, in the tail by means of the simulated annealing (SA) heuristic. SA has been found capable to provide good solutions to the problems in the OR-library (Chiyoshi and Galvao, 2000). For each problem we run 
the SA for 10,000 iterations (or 100,000 iterations for 22 test problems with a complexity of 6.95 and higher). For each problem we run SA 100 times with unique random starting values.

A statistic that turned out to be important is $S R$ given by the ratio of $1000 \sigma\left(\tilde{x}_{i}\right) / \hat{\theta}_{J K}^{(1)}$ being a measure of the similarity of the heuristic solutions and thereby potentially an indication of whether the sample is in the tail near to $\theta$ or not. Figure 2 shows $S R$ as a function (regression lines imposed) of the problems' complexity evaluated after 1,000 and 10,000 iterations and 100,000 when applicable. Some points are worth noting. A more complex, i.e. a more difficult, $p$-median problem will have a greater variation in the heuristic solutions as a consequence of many of the solutions far from the minimum. As the number of iterations increases, the $S R$ generally decreases as more and more solutions are approaching the minimum.

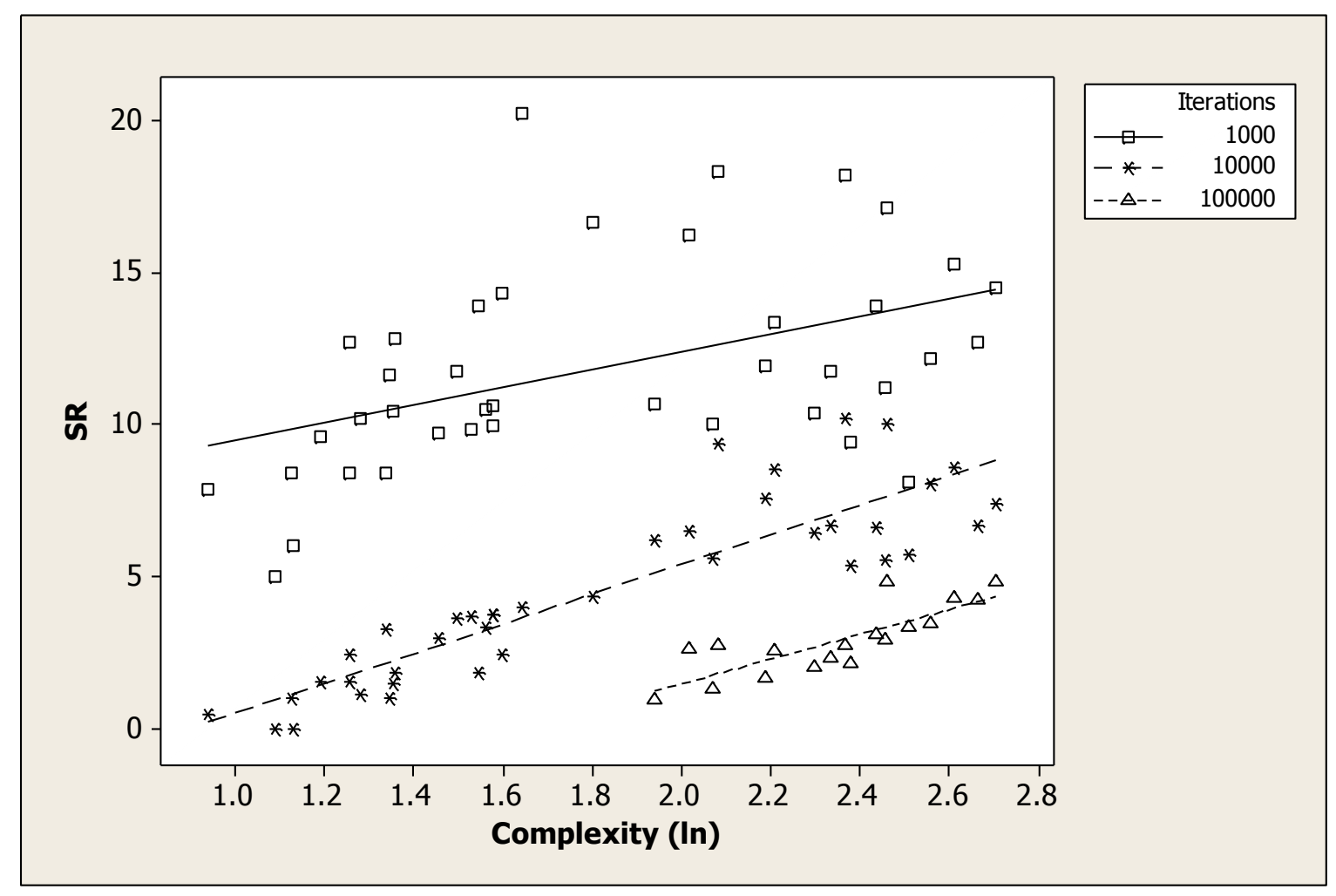

Figure 2: SR as a function of the test problems complexity.

\subsection{The bias of point-estimation}

We begin by checking if the estimators can point-estimate the minimum. Since it is computationally costly to run the heuristics 100 times for 100,000 iterations for each of the 40 test problems we only do this ones. In examining the statistical properties of the estimators we re-sample with replacement 
from the sample of heuristic solutions. We show in Table 2 some results deferring the complete listing of all problems to the Appendix. The table shows the results for the heuristic after 10,000 iterations and for $n=10,100$. It was evident that the size of $n=3$ typically made the estimators fail in estimating the minimum as well as setting statistical bounds so this case will not be thoroughly discussed in the sequel. For the three simpler problems the bias is about 0 meaning that the minimum is well estimated by all the four approaches. For the three more complex problems all approaches are biased and over-estimate the minimum to the same degree. Hence, none of the approaches seems superior in terms of estimating the minimum. The bias persists when $n=100$ is considered, indicating that there is no apparent gaining in increasing $n$ beyond 10. By increasing the iterations to 100,000 the bias of the $13^{\text {th }}$ problem is eliminated and reduced for the two most complex problems in a similar way for all estimators (see Appendix).

Table 2: Bias of the estimators evaluated after 10,000 iterations.

\begin{tabular}{|lccccccc|}
\hline \hline Problem & Complexity & $n$ & $\theta$ & $\hat{\theta}_{J K}^{(1)}-\theta$ & $\hat{\theta}_{J K}^{(2)}-\theta$ & $\hat{\theta}_{W, G}-\theta$ & $S R$ \\
\hline P11 & 2.56 & 10 & 7696 & 0 & 0 & 0 & 0.20 \\
& & 100 & & 0 & 0 & 0 & 0.44 \\
P2 & 3.88 & 10 & 4093 & 0 & 0 & 0 & 1.46 \\
& & 100 & & 0 & 0 & 0 & 1.46 \\
P36 & 4.77 & 10 & \multirow{2}{*}{9934} & 4 & 16 & 0 & 1.45 \\
& & 100 & & 1 & 1 & 1 & 3.32 \\
P13 & 6.95 & 10 & 4374 & 15 & 23 & 14 & 5.88 \\
& & 100 & & 15 & 15 & 15 & 6.19 \\
P33 & 10.81 & 10 & 4700 & 118 & 129 & 116 & 5.19 \\
& & 100 & & 115 & 115 & 117 & 5.34 \\
P30 & 14.93 & 10 & \multirow{2}{*}{1989} & 84 & 92 & 82 & 7.14 \\
& & 100 & & 80 & 81 & 82 & 7.38 \\
\hline
\end{tabular}

Although it was expected to find the Weibull and Gumbel estimators of the minimum to be positively biased, it was dejecting to find the bias of the Jackknife-estimators to be significant. However, the practical value of the estimators lies in their ability in providing intervals containing the minimum. We therefore examine the intervals' coverage percentage of the minimum $\theta$.

\subsection{Do the intervals cover the optimum?}

In Table 3 the same subset of test problems as of Table 2 are shown. We show the intervals obtained 
from the $2^{\text {nd }}$ order Jackknife and the Weibull approaches while results for the other test problems and estimators are given in the Appendix. To compute the coverage percentage of the Weibull approach for Problem 11 we took a sample with replacement of 10 (or 100) of the 100 heuristic solutions to the problem. Thereafter we computed the lower bound and checked if the minimum of 7696 was within the lower bound and the best solution (upper bound). We repeated the procedure 1,000 times and found the proportion of times the interval covered the minimum being 1.00 for this problem. The eighth column of the table gives the bounds of the intervals being the mean of the lower and the upper bounds in the 1,000 replications. The coverage and bounds for the other estimators were computed in an identical manner. With a purported confidence level of almost one for all the estimators regardless of whether $n$ equals 10 or 100, the coverage should ideally be one. This is the case for the simpler test problems, but certainly not for the more complex problems. On the contrary, for the most complex problem (P30) all of the 1,000 intervals exceeded the minimum for $n=100$.

Table 3: Bounds and coverage of Jackknife ( $2^{\text {nd }}$ order $)$ and Weibull after 10,000 iterations.

\begin{tabular}{|lcccccccc|}
\hline \hline Problem & Complexity & $n$ & $\theta$ & Coverage $^{\mathrm{JK}}$ & Interval $^{\mathrm{JK}}$ & Coverage $^{\mathrm{W}}$ & Interval $^{\mathrm{W}}$ & $S R$ \\
\hline P11 & 2.56 & 10 & 7696 & 1.00 & {$[7696,7696]$} & 1.00 & {$[7696,7696]$} & 0.20 \\
& & 100 & & 1.00 & {$[7696,7696]$} & 1.00 & {$[7696,7696]$} & 0.44 \\
P2 & \multirow{2}{*}{3.88} & 10 & 4093 & 1.00 & {$[4079,4093]$} & 1.00 & {$[4083,4093]$} & 1.46 \\
& & 100 & & 1.00 & {$[4093,4093]$} & 1.00 & {$[4081,4093]$} & 1.46 \\
P36 & 4.77 & 10 & 9934 & 0.96 & {$[9853,9950]$} & 1.00 & {$[9887,9950]$} & 1.45 \\
& & 100 & & 0.98 & {$[9908,9935]$} & 1.00 & {$[9867,9935]$} & 3.32 \\
P13 & \multirow{2}{*}{6.95} & 10 & 4374 & 0.91 & {$[4329,4397]$} & 0.99 & {$[4355,4397]$} & 5.88 \\
& & 100 & & 0.23 & {$[4374,4389]$} & 1.00 & {$[4344,4389]$} & 6.19 \\
P33 & \multirow{2}{*}{10.81} & 10 & 4700 & 0.20 & {$[4737,4829]$} & 0.03 & {$[4773,4829]$} & 5.19 \\
& & 100 & & 0.00 & {$[4793,4817]$} & 0.00 & {$[4758,4817]$} & 5.34 \\
P30 & \multirow{2}{*}{14.93} & 10 & \multirow{2}{*}{1989} & 0.19 & {$[2019,2081]$} & 0.03 & {$[2047,2081]$} & 7.14 \\
& & 100 & & 0.00 & {$[2051,2071]$} & 0.00 & {$[2034,2071]$} & 7.38 \\
\hline
\end{tabular}

It is reasonable to infer that $\tilde{x}_{i}$ will converge when the solutions come close to the optimum, and consequently the standard deviation of them would also decrease. By dividing the standard deviation of $\tilde{x}_{i}$ by $\hat{\theta}_{J K}^{(1)}$ we get a measure of similarity amongst the solutions and thereby how far out in the tail and how close to the optimum the solutions are to be (reported in Tables 2-3 as column SR). The first three problems all have small SR. Correspondingly, the bias of the 
point-estimators is small, and the coverage of the intervals' are close to 1. For the last three problems being more complex SR and the bias is large and the coverage percentage is poor. Hence, a large SR indicates that the $\tilde{x}_{i}$ :s are not sufficiently near to the optimum for a reliable estimation of the minimum and bounds of it. Additional iterations of the heuristic might improve the situation.

We proceed by increasing the iterations for problems P13 to P30 having SR greater than 5. Table 4 gives again the coverage and the bounds of Weibull and the $2^{\text {nd }}$ order Jackknife approach where the procedure is identical to the one described in relation to Table 3 with the exception that the number of iterations is 100,000 .

Table 4: Bounds and coverage of Jackknife ( $2^{\text {nd }}$ order) and Weibull after 100,000 iterations.

\begin{tabular}{|lcccccccc|}
\hline \hline Problem & Complexity & $n$ & $\theta$ & Coverage $^{\text {JK }}$ & Interval $^{\mathrm{JK}}$ & Coverage $^{\mathrm{W}}$ & Interval $^{\mathrm{W}}$ & $S R$ \\
\hline P13 & 6.95 & 10 & 4374 & 1.00 & {$[4370,4374]$} & 1.00 & {$[4370,4374]$} & 0.91 \\
& & 100 & & 1.00 & {$[4374,4374]$} & 1.00 & {$[4369,4374]$} & 0.93 \\
P33 & 10.81 & 10 & \multirow{2}{*}{4700} & 0.84 & {$[4681,4714]$} & 0.99 & {$[4694,4714]$} & 2.07 \\
& & 100 & & 0.80 & {$[4690,4707]$} & 1.00 & {$[4682,4707]$} & 2.12 \\
P30 & 14.93 & 10 & \multirow{2}{*}{1989} & 0.49 & {$[1982,2018]$} & 0.44 & {$[1995,2018]$} & 4.71 \\
& & 100 & & 0.28 & {$[1990,2009]$} & 0.96 & {$[1984,2009]$} & 4.83 \\
\hline
\end{tabular}

The problems P13 and P33 have a value of SR clearly below 5 after 100,000 iterations (Table 4). As a result the intervals generally cover the minimum. The large number of iterations is, on the other hand, possibly insufficient for the most complex problem (P30) as SR is about 5 and has intervals that often fail to cover the actual minimum.

\subsection{The coverage and $S R$}

An examination of the intervals' coverage for a few test problems is insufficient for drawing any general conclusions about the relationship between coverage and SR. Figure 3 shows the coverage as a function of SR for all four estimators and $n=100$. The first thing to note is that the coverage falls drastically from about the nominal level of one to about 0.5 around SR being 4 and higher. For SR below both the Weibull and the Gumbel estimator have excellent coverage whereas the two Jackknife estimators are less reliable with the first order Jackknife-estimator being the worst. Lastly, the functions depicted in the figure were estimated based on all the experiments and on all levels of iterations being 1,000,10,000 and (for about half of the test problems) 100,000. In estimating the 
functions we did not find any indication of different patterns depending on the number of iterations. Hence we think the results can be interpolated for iterations in the range of 1,000 to 100,000 , and probably also extrapolated beyond 100,000 iterations.

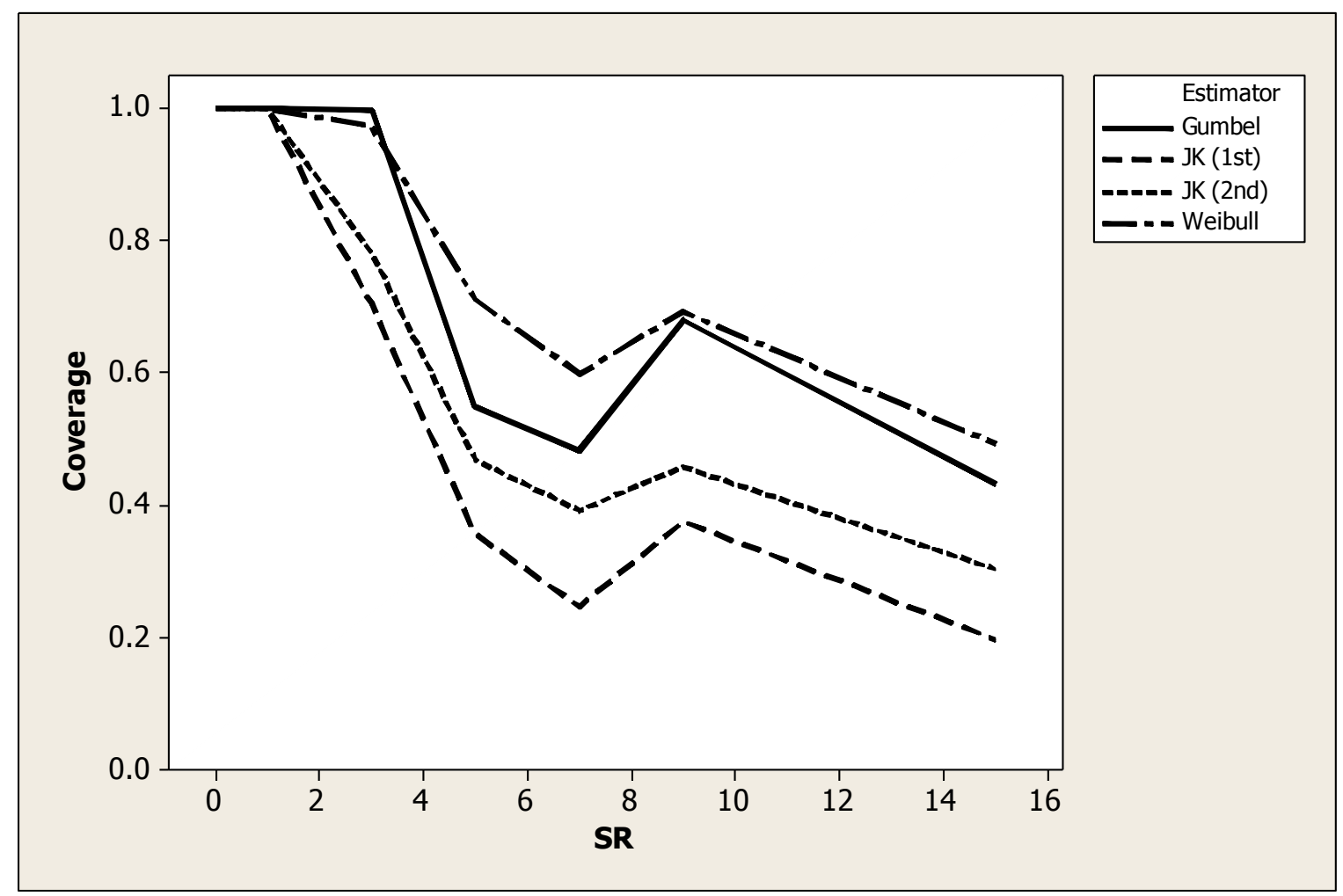

Figure 3: Coverage as a function of SR, $n=100$ 


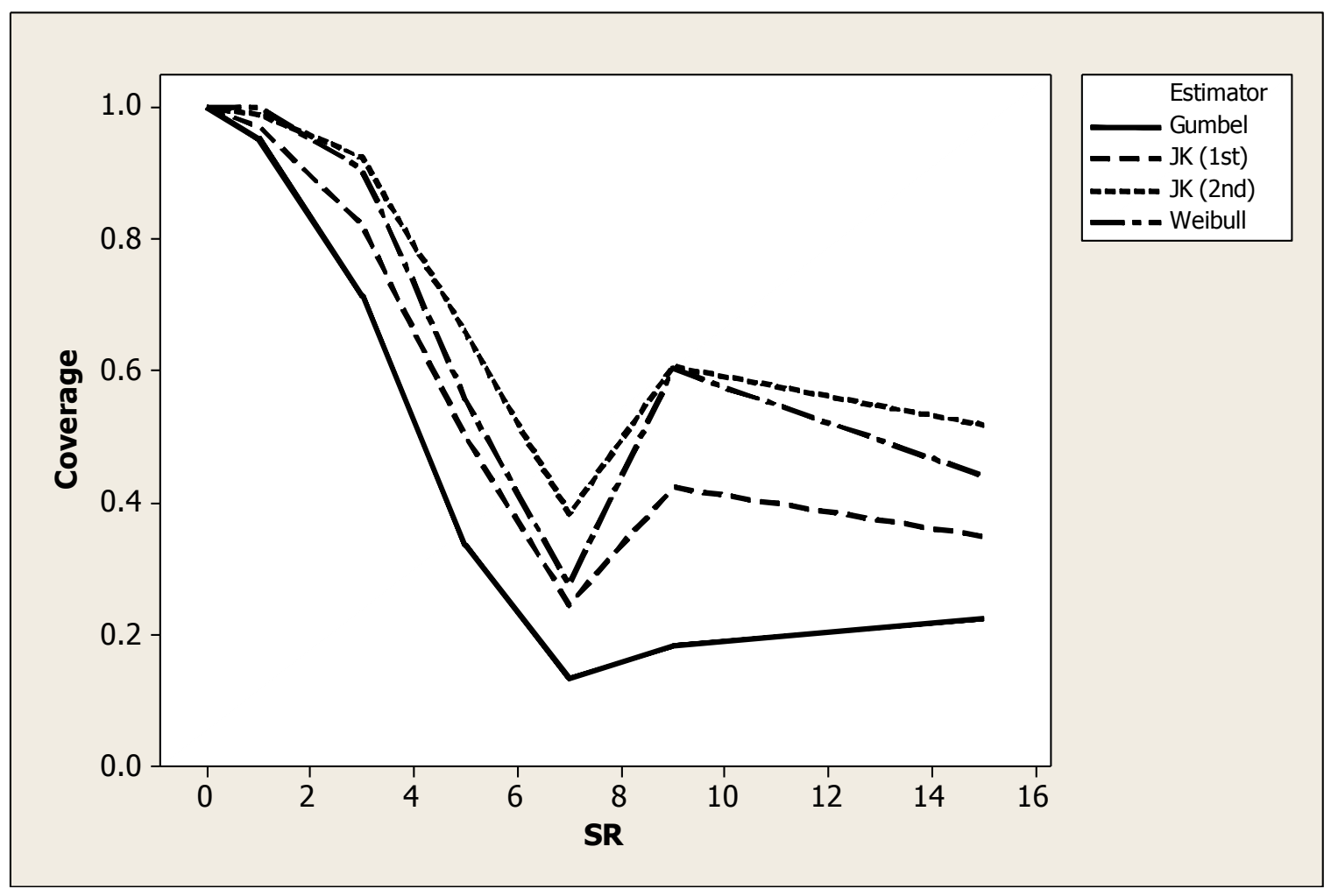

Figure 4: Coverage as a function of SR, $n=10$

However, running the heuristic algorithm in 100 parallel processes is computationally costly and it is therefore worthwhile to check how the estimators manage to uncover the minimum using a smaller sample of heuristic solutions. In Figures 4 and 5 we show the coverage as a function of SR for the two lower levels of $n$. Evident from the two figures is that the Gumbel estimator is quite poor if its parameters are estimated on 10 or less observations. The Weibull estimators works well in the case of $n=10$. The second order Jackknife estimator is the best estimator for $n \leq 10$ and gives decent coverage even in the case of $n=3$ as long as SR is below 4 . 


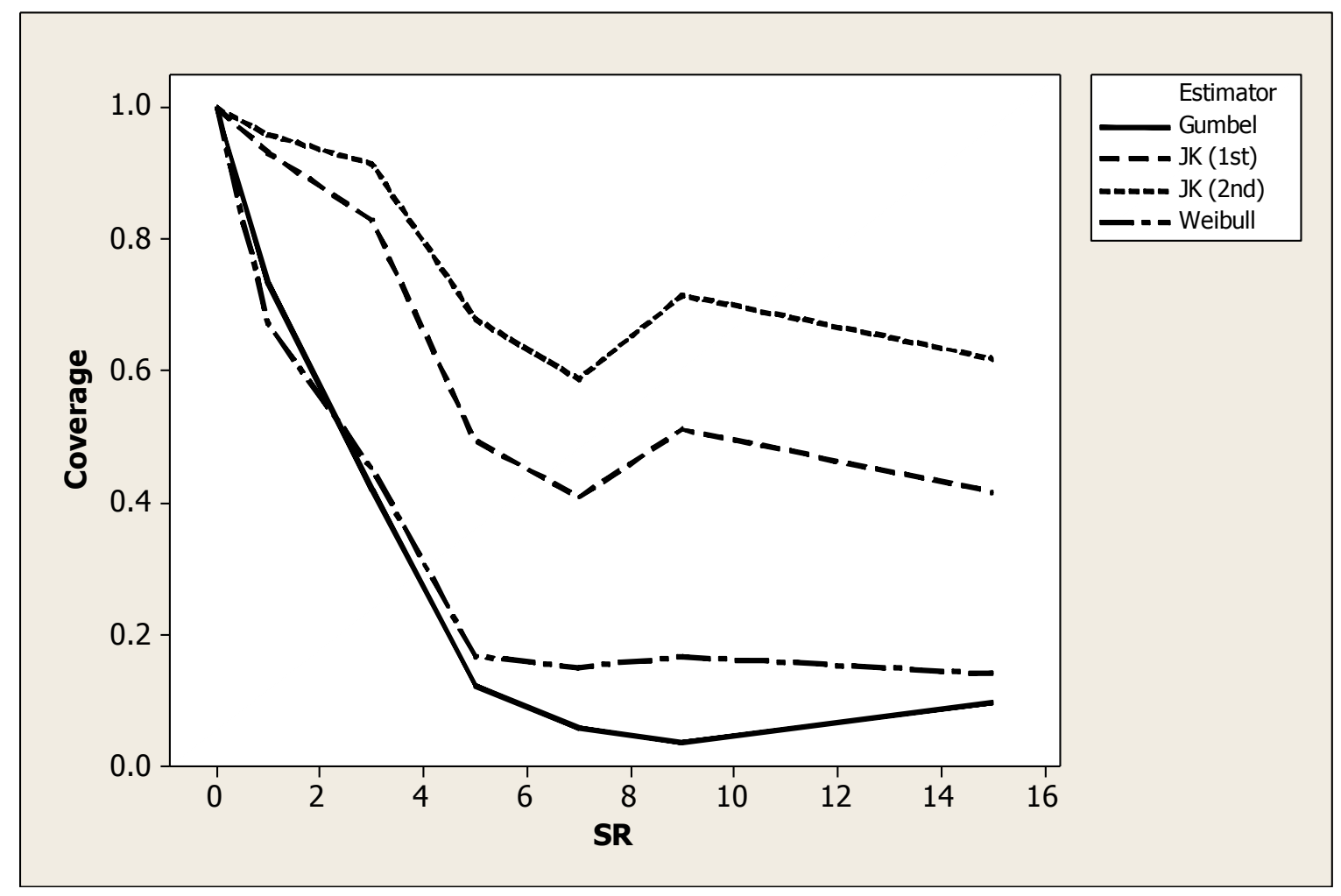

Figure 5: Coverage as a function of SR, $n=3$

To conclude the results that draws on our extensive experimentation with the 40 test problems from the OR-library. The Gumbel estimators and the first order Jackknife estimators are inferior to the alternatives. A sample of $n=10$ seems sufficient for a reliable estimation of statistical bounds for the minimum, given that SR is modest (around 4 or less). If the SR is large, then no estimator provides reliable bounds.

In the Appendix there are complete tables giving all details for all the test problems, including information about the length of the intervals.

\section{An illustrating problem}

In this section, we illustrate the estimators applied to a practical location problem concerning allocating several distribution centers of the Swedish Post in one region in Sweden. In this real location problem the minimum is unknown. The problem is to allocate 71 distribution centers of the Swedish Post on some of the 6,735 candidate nodes in the network of Dalarna in mid-Sweden. The landscape of the region and its population of 277,725 inhabitants, distributed in 15729 demand 
points, is described by Carling, Han, and Håkansson (2012). Han, Håkansson, and Rebreyend (2013) provides a detailed description of the road network and urge that network distance is used as distance measure. So we take the objective function as the average distance in meters on the road network to the nearest postal center for the population.

To appreciate the complexity of this real-world problem, we provide the distribution of the objective function. As we did for the OR-lib problems, we draw a random sample of 1,000,000 and show the empirical distribution of $g\left(z_{p}\right)$ in Figure 6. The distribution is slightly skewed to the right, but still approximately Normal. To evaluate the complexity of the problem, we use $\hat{\theta}_{J K}^{(2)}$ as the estimate of the minimum $\theta$, and the mean and variance of $g\left(z_{p}\right)$ are derived by the 1 million random sample. The complexity is 5.47 which is in the middle of the 40 OR-lib problems.

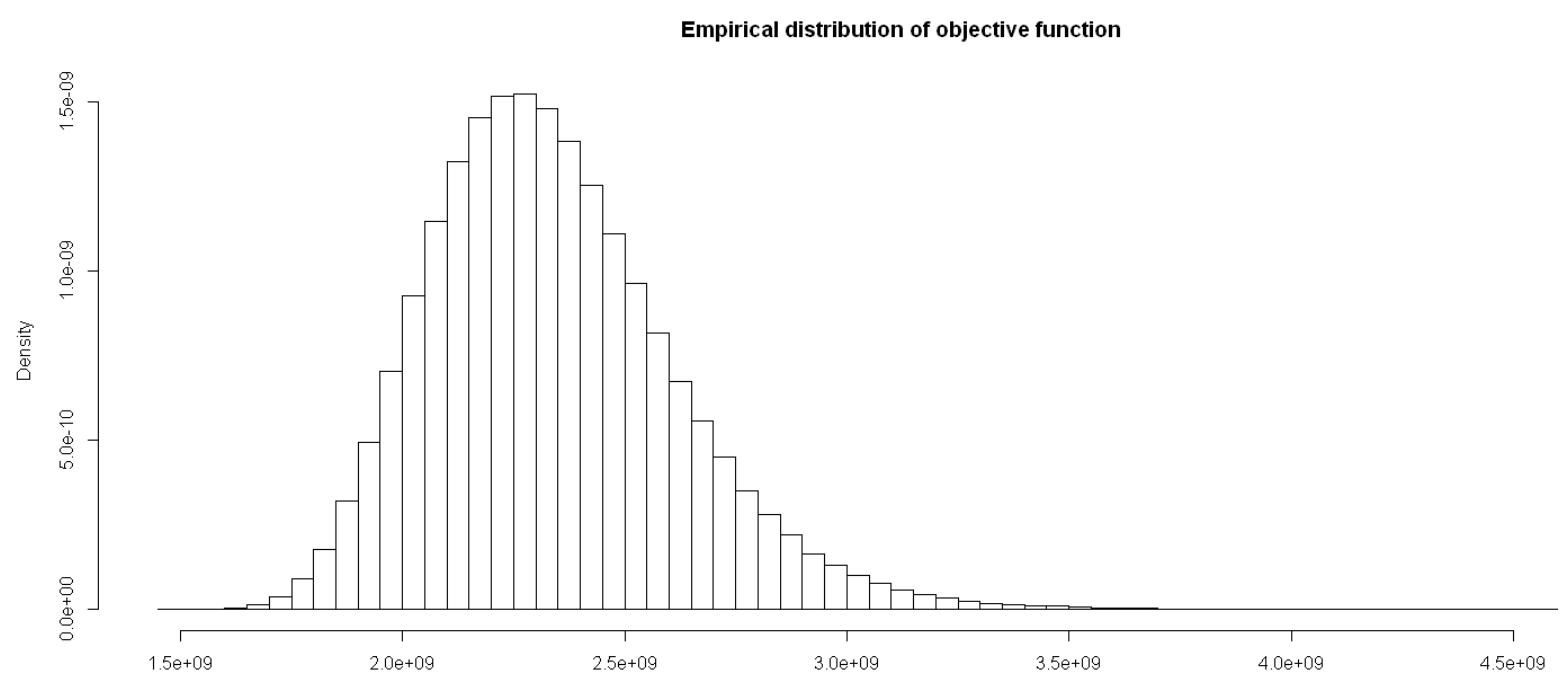

Figure 6: Empirical distribution of objective function for the Swedish Post problem.

Drawing on the experimental results above, we set $n=10$ and run the heuristic algorithm for 10,000 iterations. We intended to run the algorithm until SR converged to 4 or less. We checked SR after every step of 10,000 iterations and Figure 7 shows the evolution of SR (as well as the best heuristic solution and the Jackknife estimator of the minimum) as a function of the number of iterations. As can be seen in the figure, at about 300,000 iterations SR was equal to 3.12 and since the statistical bounds were quite tight we decided to stop the heuristic. 


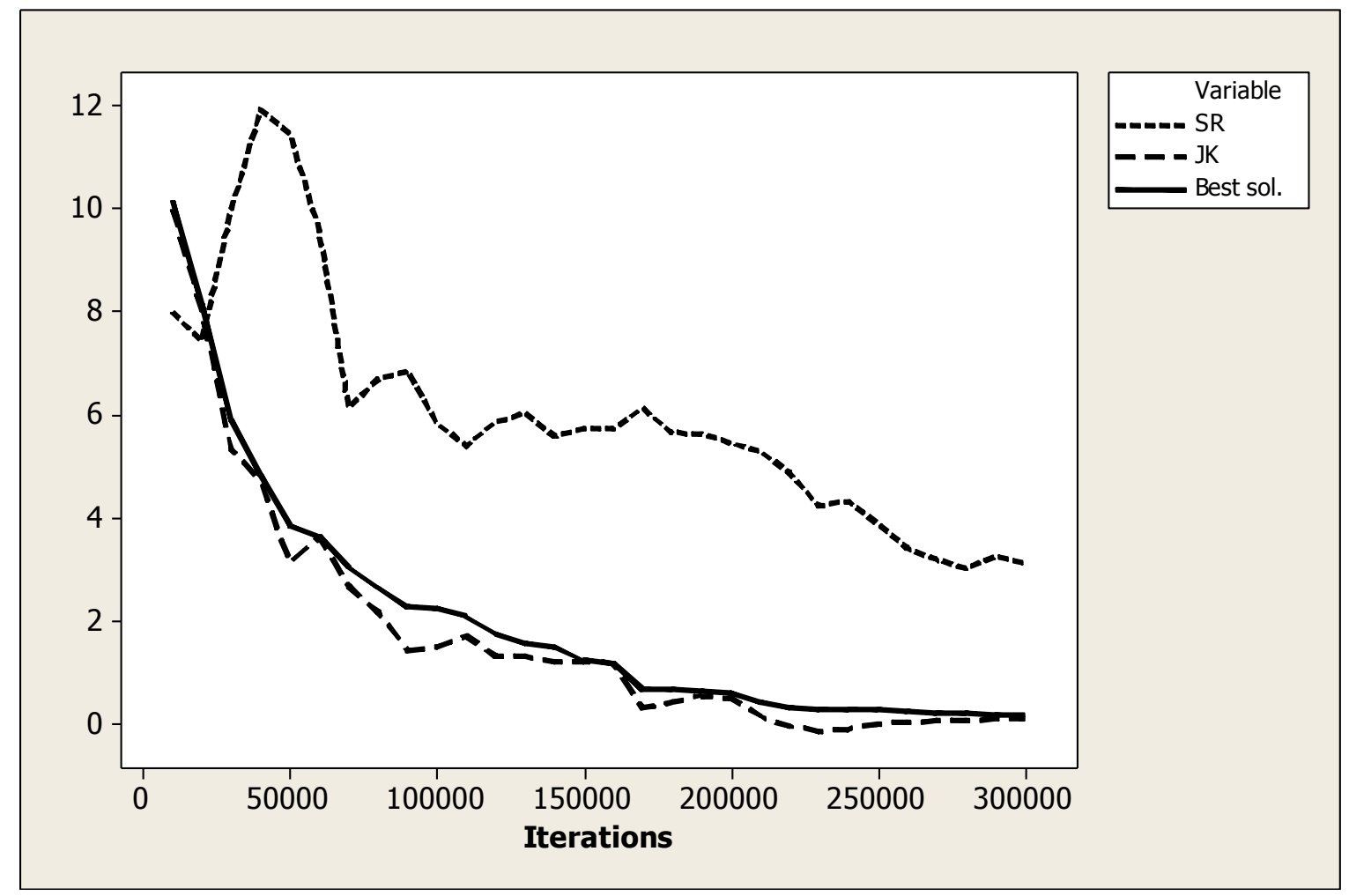

Figure 7: The Swedish Post problem and SR by the number of iterations. The best solution (\%) and the Jackknife $\left(1^{\text {st }}\right.$ order) point-estimate $(\%)$ relative to the best solution after 300,000 iterations.

Upon stopping the heuristic algorithm we used the sample of the 10 heuristic solutions to compute statistical bounds for the minimum using all the four estimators. The point estimators were $\hat{\theta}_{J K}^{(1)}=2973, \hat{\theta}_{J K}^{(2)}=2972$, and $\hat{\theta}_{W, G}=2975$ with the latter being the upper bound. The lower bounds were [2964, 2956, 2959, 2966] for first and second Jackknife, Weibull, and Gumbel estimators, respectively. Hence, all estimators suggest an interval of at the most 20 meters which is a tolerable error for this application.

\section{Concluding discussion}

We have considered the problem of knowing when a solution provided by a heuristic is close to optimal. Deterministic bounds may sometimes be applicable and enough tight to shed knowledge on the problem. We have, however, studied statistical bounds potentially being of a more general applicability. We have studied the occasionally used Weibull estimator as well as two variants on the 
Jackknife estimator which, to our knowledge, never has been used for obtaining bounds in location problems. Furthermore, we have argued for and examined an alternative EVT estimator being the Gumbel estimator.

Derigs (1985) made a number of concluding observations upon studying statistical bounds with regard to TSP and QAP. We think that most of his conclusions are still valid, except one. Derigs stated "The Weibull approach leads to a proper approach,". We have however demonstrated that none of the estimators, including Weibull, are reliable unless the quality of the sample of heuristic solutions used for deriving the bounds is of high quality. To assess the quality we suggest using SR which is the standard deviation of the $n$ solutions divided by the Jackknife point-estimator. The experiments suggest that SR exceeding 4 causes unreliable bounds.

The estimators performed similarly with a slight advantage for the second order Jackknife and the Weibull estimator. In fact, if one cannot afford to run many processes in parallel to get large $n$, the second order Jackknife is the first choice. We did address the question of the size of $n$. There is not much research on the size of $n$, but previous researchers have indicated that $n$ needs to be at least 100. Our results suggest this size to be overly pessimistic, in fact most estimators provided reliable bounds at $n$ equal to 10 (and the second order Jackknife provided fairly reliable bounds even at $n$ equal to 3$)$.

We have limited the study to location problems by means of the $p$-median problems in the OR-library for which the optimum is known, and a real world $p$-median problem. It seems that $g\left(z_{p}\right)$ closely follows the Normal distribution in these cases. Other combinatorial problems may imply, from a statistical perspective, objective functions of a more complicated kind such as multi-modal or skewed distributions. Moreover, our results seem to hold for all of the OR-lib problems that represents a substantial variation in kind and complexity. However, we consistently used simulated annealing as the heuristic. We do not think this choice is crucial for our findings since the heuristic only serves to obtain a sample in the tail of the distribution and any heuristic meeting this requirement should work. However, it goes without saying that extending the variation in combinatorial problems and heuristic algorithms used would expand the knowledge about the value of statistical bounds in combinatorial optimization. 


\section{Acknowledgement}

We are grateful to participants at INFORMS Euro 2013 in Rome for useful comments on an earlier version. Financial support from the Swedish Retail and Wholesale Development Council is gratefully acknowledged.

\section{References}

Akyüz, M.H., Öncan, T., Altınel, I.K., (2012). Efficient approximate solution methods for the multi-commodity capacitated multi-facility Weber problem. Computers \& Operations Research 39.2, 225-237.

Beasley, J.E., (1990), OR library: Distributing test problems by electronic mail, Journal of Operational Research Society, 41:11, 1067-1072.

Beasley, J.E., (1993). Lagrangian heuristics for location problems. European Journal of Operational Research, 65, 383-399.

Carling, K., Han, M., Håkansson, J., (2012). Does Euclidean distance work well when the p-median model is applied in rural areas? Annals of Operations Research, 201:1, 83-97.

Chiyoshi, F.Y., and Galvão, R.D., (2000). "A statistical analysis of simulated annealing applied to the p-median problem”, Annals of Operations Research 96, 61-74.

Dannenbring, D.G., (1977), Procedures for estimating optimal solution values for large combinatorial problems, Management science, 23:12, 1273-1283.

Daskin, M.S., (1995). Network and discrete location: models, algorithms, and applications. New York: Wiley.

Derigs, U, (1985). Using confidence limits for the global optimum in combinatorial optimization. Operations research, 33:5, 1024-1049.

Efron, B., (1979). Bootstrap methods: Another look at the Jackknife. Annals of statistics, 7:1, 1-26.

Golden, B.L., Alt, F.B., 1979. Interval estimation of a global optimum for large combinatorial optimization, Operations Research 33:5, 1024-1049.

Hakimi, S.L., (1964). Optimum locations of switching centers and the absolute centers and medians of a graph, Operations Research, 12:3, 450-459.

Hakimi, S.L., (1965). Optimum Distribution of Switching Centers in a Communication Network and Some Related Graph Theoretic Problems, Operations Research, 13:3, 462-475. 
Han, M., Håkansson, J., Rebreyend, P. (2013). How do different densities in a network affect the optimal location of service centers?, Working papers in transport, tourism, information technology and microdata analysis, 2013:15.

Handler, G.Y., and Mirchandani, P.B., (1979). Location on networks: Theorem and algorithms, MIT Press, Cambridge, MA.

Kotz, S., and Nadarajah, S., (2000). Extreme value distributions, theory and applications. Imperial College Press.

Levanova, T., and Loresh, M.A., (2004). Algorithm of ant system and simulated annealing for the p-median problem, Automation and Remote Control 65, 431-438.

Luis, M., Sahli, S., Nagy, G., (2009). Region-rejection based heuristics for the capacitated multi-source Weber problem. Computers \& Operations Research 36, 2007-2017.

McRobert, K.L., 1971. A search model for evaluating combinatorially explosive problems, Operations Research, 19, 1331-1349.

Nydick JR, R.L., and Weiss, H.J., (1988), A computational evaluation of optimal solution value estimation procedures, Computers \& Operations Research, 5, 427-440.

Quenouille, M.H., (1956), Notes on bias in estimation, Biometrika, 43, 353-360.

Robson, D.S., and Whitlock, J.H., (1964), Estimation of a truncation point, Biometrika, 51, 33-39.

Wilson, A.D., King, R.E., and Wilson, J.R., (2004), Case study on statistically estimating minimum makespan for flow line scheduling problems, European Journal of Operational Research, 155, 439-454. 


\section{Appendix}

Table A1: Description of the other 34 problems of the OR-library.

\begin{tabular}{|lcccc|}
\hline \hline Problem & $\theta$ & $\mu_{g\left(z_{p}\right)}$ & $\sigma_{g\left(z_{p}\right)}$ & Complexity \\
\hline P1 & 5819 & 8426 & 877 & 2.97 \\
P16 & 8162 & 11353 & 1033 & 3.09 \\
P6 & 7824 & 10522 & 869 & 3.10 \\
P26 & 9917 & 13644 & 1133 & 3.29 \\
P21 & 9138 & 12906 & 1067 & 3.52 \\
P38 & 11060 & 15078 & 1143 & 3.52 \\
P31 & 10086 & 13960 & 1077 & 3.60 \\
P35 & 10400 & 14179 & 1085 & 3.81 \\
P7 & 5631 & 7930 & 598 & 3.84 \\
P3 & 4250 & 6194 & 500 & 3.89 \\
P27 & 8307 & 11428 & 727 & 4.29 \\
P17 & 6999 & 9819 & 631 & 4.47 \\
P22 & 8579 & 11699 & 676 & 4.62 \\
P12 & 6634 & 9387 & 586 & 4.70 \\
P39 & 9423 & 12988 & 736 & 4.84 \\
P32 & 9297 & 12687 & 699 & 4.85 \\
P4 & 3034 & 4618 & 320 & 4.95 \\
P5 & 1355 & 2376 & 197 & 5.18 \\
P8 & 4445 & 6604 & 356 & 6.07 \\
P9 & 2734 & 4250 & 202 & 7.51 \\
P18 & 4809 & 6769 & 248 & 7.92 \\
P10 & 1255 & 2278 & 127 & 8.02 \\
P23 & 4619 & 6586 & 220 & 8.94 \\
P14 & 2968 & 4501 & 168 & 9.12 \\
P28 & 4498 & 6369 & 188 & 9.95 \\
P19 & 2845 & 4327 & 144 & 10.32 \\
P15 & 1729 & 2896 & 109 & 10.67 \\
P24 & 2961 & 4486 & 134 & 11.42 \\
P37 & 5057 & 7246 & 188 & 11.65 \\
P20 & 1789 & 3108 & 112 & 11.73 \\
P40 & 5128 & 7329 & 179 & 12.32 \\
P29 & 3033 & 4559 & 118 & 12.93 \\
P25 & 1828 & 3131 & 95 & 13.64 \\
P34 & & 4617 & 112 & 14.36 \\
\hline
\end{tabular}



Table A2: Results for the estimators in the computer experiments. $n=3$

\begin{tabular}{|c|c|c|c|c|c|c|c|c|c|c|c|c|c|}
\hline \multirow[b]{2}{*}{ Problem } & \multirow[b]{2}{*}{ Iter. } & \multirow[b]{2}{*}{$S R$} & \multicolumn{3}{|c|}{$\mathrm{JK}\left(1^{\mathrm{st}}\right)$} & \multicolumn{3}{|c|}{$\mathrm{JK}\left(2^{\mathrm{nd}}\right)$} & \multicolumn{2}{|c|}{ Weibull } & \multirow{2}{*}{$\begin{array}{c}\hat{\theta}_{W, G} \\
\text { Bias }\end{array}$} & \multicolumn{2}{|c|}{ Gumbel } \\
\hline & & & Bias & Cov. & Length & Bias & Cov. & Length & Cov. & Length & & Cov. & Length \\
\hline P11 & 1000 & 6.8 & -1 & 0.93 & 184 & 19 & 0.97 & 336 & 0.64 & 0 & 35 & 0.35 & 23 \\
\hline P11 & 10000 & 0.1 & 0 & 1.00 & 2 & 1 & 1.00 & 3 & 1.00 & 0 & 0 & 1.00 & 1 \\
\hline P1 & 1000 & 4.1 & -10 & 0.99 & 81 & 9 & 0.99 & 142 & 0.77 & 0 & 3 & 0.92 & 12 \\
\hline P1 & 10000 & 0.0 & 0 & 1.00 & 0 & 0 & 1.00 & 0 & 1.00 & 0 & 0 & 1.00 & 0 \\
\hline P16 & 1000 & 7.6 & -7 & 0.93 & 222 & 20 & 0.97 & 387 & 0.61 & 0 & 38 & 0.39 & 26 \\
\hline P16 & 10000 & 0.7 & -2 & 1.00 & 17 & 5 & 1.00 & 31 & 0.92 & 0 & 0 & 0.98 & 4 \\
\hline P6 & 1000 & 5.2 & -9 & 0.92 & 151 & 3 & 0.97 & 264 & 0.62 & 9 & 23 & 0.47 & 16 \\
\hline P6 & 10000 & 0.0 & 0 & 1.00 & 0 & 0 & 1.00 & 0 & 1.00 & 0 & 0 & 1.00 & 0 \\
\hline P26 & 1000 & 8.9 & 40 & 0.81 & 355 & 42 & 0.92 & 621 & 0.44 & 0 & 123 & 0.10 & 29 \\
\hline P26 & 10000 & 0.9 & -2 & 0.97 & 25 & 8 & 0.97 & 44 & 0.98 & 7 & 0 & 0.95 & 6 \\
\hline P21 & 1000 & 11.5 & 33 & 0.85 & 416 & 42 & 0.94 & 726 & 0.57 & 0 & 128 & 0.13 & 40 \\
\hline P38 & 1000 & 7.5 & 69 & 0.78 & 333 & 74 & 0.91 & 580 & 0.30 & 0 & 146 & 0.04 & 29 \\
\hline P21 & 10000 & 0.7 & 0 & 1.00 & 16 & 10 & 1.00 & 29 & 0.97 & 0 & 0 & 1.00 & 4 \\
\hline P38 & 10000 & 2.0 & -10 & 0.97 & 77 & 6 & 0.98 & 135 & 0.72 & 0 & 3 & 0.87 & 11 \\
\hline P31 & 1000 & 8.9 & 40 & 0.83 & 338 & 64 & 0.94 & 589 & 0.55 & 12 & 113 & 0.14 & 34 \\
\hline P31 & 10000 & 0.5 & -1 & 0.94 & 15 & 6 & 0.94 & 27 & 0.94 & 0 & 0 & 0.92 & 3 \\
\hline P35 & 1000 & 7.5 & 69 & 0.78 & 333 & 98 & 0.92 & 636 & 0.26 & 43 & 146 & 0.02 & 36 \\
\hline P35 & 10000 & 2.1 & -5 & 1.00 & 62 & 22 & 1.00 & 110 & 0.95 & 0 & 1 & 0.99 & 14 \\
\hline P7 & 1000 & 9.8 & 40 & 0.80 & 198 & 64 & 0.92 & 345 & 0.37 & 29 & 80 & 0.08 & 25 \\
\hline P7 & 10000 & 0.9 & -2 & 0.93 & 20 & -1 & 0.95 & 34 & 0.77 & 0 & 2 & 0.82 & 2 \\
\hline P2 & 1000 & 9.4 & 2 & 0.92 & 141 & 15 & 0.97 & 246 & 0.62 & 17 & 32 & 0.26 & 15 \\
\hline P2 & 10000 & 1.2 & -3 & 0.84 & 23 & -4 & 0.84 & 41 & 1.00 & 0 & 2 & 0.87 & 2 \\
\hline P3 & 1000 & 11.1 & -1 & 0.90 & 174 & 15 & 0.96 & 303 & 0.63 & 11 & 35 & 0.35 & 20 \\
\hline P3 & 10000 & 0.9 & 0 & 1.00 & 10 & 6 & 1.00 & 17 & 0.96 & 0 & 0 & 1.00 & 2 \\
\hline P27 & 1000 & 8.6 & 114 & 0.67 & 286 & 119 & 0.85 & 499 & 0.22 & 48 & 180 & 0.00 & 27 \\
\hline P27 & 10000 & 2.5 & -4 & 0.95 & 75 & 5 & 0.98 & 131 & 0.63 & 4 & 11 & 0.51 & 9 \\
\hline P17 & 1000 & 10.5 & 105 & 0.67 & 294 & 111 & 0.86 & 513 & 0.17 & 0 & 172 & 1.00 & 14 \\
\hline P17 & 10000 & 2.8 & -5 & 0.97 & 63 & 9 & 0.99 & 111 & 0.77 & 5 & 6 & 1.00 & 8 \\
\hline P22 & 1000 & 8.5 & 162 & 0.56 & 280 & 182 & 0.80 & 487 & 0.18 & 0 & 223 & 0.00 & 31 \\
\hline P22 & 10000 & 3.2 & -10 & 0.96 & 100 & 1 & 0.98 & 175 & 0.66 & 10 & 10 & 0.60 & 12 \\
\hline P12 & 1000 & 12.2 & 48 & 0.83 & 315 & 61 & 0.92 & 549 & 0.36 & 39 & 119 & 0.08 & 33 \\
\hline P12 & 10000 & 1.2 & -2 & 0.99 & 24 & 8 & 1.00 & 42 & 0.92 & 3 & 0 & 0.98 & 5 \\
\hline P36 & 1000 & 9.4 & 173 & 0.61 & 354 & 199 & 0.82 & 617 & 0.19 & 52 & 250 & 0.00 & 35 \\
\hline P36 & 10000 & 2.9 & 9 & 0.86 & 108 & 17 & 0.93 & 188 & 0.60 & 7 & 33 & 0.17 & 11 \\
\hline P39 & 1000 & 8.7 & 192 & 0.57 & 340 & 190 & 0.78 & 593 & 0.15 & 0 & 272 & 0.00 & 29 \\
\hline P39 & 10000 & 3.3 & 3 & 0.90 & 120 & 8 & 0.95 & 209 & 0.56 & 3 & 29 & 0.24 & 12 \\
\hline P32 & 1000 & 9.5 & 152 & 0.67 & 353 & 163 & 0.85 & 615 & 0.17 & 27 & 232 & 0.00 & 34 \\
\hline P32 & 10000 & 3.4 & -8 & 0.96 & 114 & 5 & 0.99 & 200 & 0.67 & 7 & 15 & 0.48 & 13 \\
\hline P4 & 1000 & 12.7 & 57 & 0.70 & 156 & 60 & 0.86 & 272 & 0.19 & 0 & 93 & 0.01 & 14 \\
\hline P4 & 10000 & 2.0 & -3 & 0.96 & 21 & 1 & 0.97 & 37 & 0.86 & 1 & 1 & 0.83 & 3 \\
\hline P5 & 1000 & 17.3 & 36 & 0.69 & 93 & 39 & 0.86 & 163 & 0.19 & 0 & 57 & 0.00 & 9 \\
\hline P5 & 10000 & 3.2 & -2 & 0.97 & 14 & 2 & 0.98 & 25 & 0.87 & 1 & 1 & 0.87 & 2 \\
\hline P8 & 1000 & 14.6 & 129 & 0.59 & 272 & 127 & 0.79 & 474 & 0.15 & 9 & 193 & 0.00 & 23 \\
\hline P8 & 10000 & 3.6 & -4 & 0.96 & 57 & 3 & 0.99 & 100 & 0.68 & 3 & 7 & 0.54 & 7 \\
\hline P13 & 1000 & 9.9 & 192 & 0.28 & 179 & 195 & 0.61 & 314 & 0.06 & 0 & 233 & 0.00 & 16 \\
\hline P13 & 10000 & 5.3 & 17 & 0.80 & 87 & 23 & 0.91 & 151 & 0.26 & 4 & 36 & 0.06 & 10 \\
\hline P13 & 100000 & 0.8 & -2 & 0.97 & 12 & 0 & 0.97 & 22 & 0.90 & 0 & 0 & 0.94 & 2 \\
\hline P9 & 1000 & 14.2 & 123 & 0.45 & 160 & 126 & 0.70 & 280 & 0.08 & 7 & 160 & 0.00 & 14 \\
\hline P9 & 10000 & 5.8 & 8 & 0.82 & 62 & 10 & 0.92 & 109 & 0.31 & 1 & 22 & 0.08 & 6 \\
\hline
\end{tabular}




\begin{tabular}{|c|c|c|c|c|c|c|c|c|c|c|c|c|c|}
\hline P9 & 100000 & 2.4 & -2 & 0.87 & 29 & -3 & 0.92 & 50 & 0.61 & 0 & 5 & 0.43 & 2 \\
\hline P18 & 1000 & 8.9 & 233 & 0.21 & 178 & 236 & 0.48 & 310 & 0.06 & 10 & 274 & 0.00 & 16 \\
\hline P18 & 10000 & 4.9 & 52 & 0.59 & 91 & 57 & 0.79 & 159 & 0.17 & 10 & 72 & 0.00 & 9 \\
\hline P18 & 100000 & 0.9 & 0 & 0.88 & 15 & 3 & 0.93 & 27 & 0.89 & 1 & 3 & 0.35 & 2 \\
\hline P10 & 1000 & 16.4 & 102 & 0.24 & 87 & 104 & 0.56 & 151 & 0.06 & 1 & 122 & 0.00 & 8 \\
\hline P10 & 10000 & 8.1 & 16 & 0.67 & 39 & 19 & 0.86 & 67 & 0.30 & 0 & 24 & 0.01 & 4 \\
\hline P10 & 100000 & 2.4 & -1 & 0.97 & 10 & 0 & 0.98 & 18 & 0.78 & 0 & 0 & 0.88 & 1 \\
\hline P23 & 1000 & 10.6 & 298 & 0.14 & 195 & 312 & 0.44 & 340 & 0.05 & 21 & 341 & 0.00 & 20 \\
\hline P23 & 10000 & 6.7 & 77 & 0.51 & 121 & 83 & 0.74 & 211 & 0.15 & 12 & 104 & 0.00 & 12 \\
\hline P23 & 100000 & 1.4 & 0 & 0.91 & 24 & 2 & 0.96 & 42 & 0.63 & 1 & 5 & 0.32 & 3 \\
\hline P14 & 1000 & 12.1 & 210 & 0.19 & 152 & 212 & 0.46 & 265 & 0.05 & 4 & 245 & 0.00 & 14 \\
\hline P14 & 10000 & 7.8 & 45 & 0.60 & 93 & 47 & 0.81 & 162 & 0.14 & 1 & 67 & 0.00 & 8 \\
\hline P14 & 100000 & 2.0 & -1 & 0.96 & 20 & 3 & 0.98 & 34 & 0.79 & 2 & 2 & 0.59 & 3 \\
\hline P28 & 1000 & 9.2 & 306 & 0.09 & 177 & 307 & 0.37 & 308 & 0.05 & 11 & 347 & 0.00 & 15 \\
\hline P28 & 10000 & 5.5 & 85 & 0.40 & 105 & 83 & 0.65 & 184 & 0.12 & 6 & 110 & 0.00 & 9 \\
\hline P28 & 100000 & 1.8 & 4 & 0.83 & 30 & 6 & 0.92 & 52 & 0.50 & 2 & 10 & 0.13 & 3 \\
\hline P19 & 1000 & 10.6 & 248 & 0.07 & 129 & 251 & 0.32 & 225 & 0.03 & 0 & 277 & 0.00 & 12 \\
\hline P19 & 10000 & 6.0 & 73 & 0.33 & 69 & 75 & 0.60 & 120 & 0.08 & 8 & 88 & 0.00 & 6 \\
\hline P19 & 100000 & 2.0 & 4 & 0.82 & 20 & 7 & 0.92 & 34 & 0.25 & 2 & 8 & 0.07 & 3 \\
\hline P15 & 1000 & 15.9 & 177 & 0.16 & 120 & 178 & 0.45 & 210 & 0.04 & 0 & 205 & 0.00 & 11 \\
\hline P15 & 10000 & 9.2 & 39 & 0.53 & 64 & 41 & 0.79 & 111 & 0.13 & 0 & 54 & 0.00 & 6 \\
\hline P15 & 100000 & 2.4 & 1 & 0.89 & 15 & 2 & 0.95 & 27 & 0.51 & 1 & 4 & 0.21 & 2 \\
\hline P33 & 1000 & 8.4 & 381 & 0.04 & 170 & 382 & 0.24 & 296 & 0.04 & 0 & 420 & 0.00 & 14 \\
\hline P33 & 10000 & 4.8 & 125 & 0.21 & 93 & 124 & 0.51 & 163 & 0.05 & 4 & 147 & 0.00 & 8 \\
\hline P33 & 100000 & 1.9 & 11 & 0.73 & 35 & 12 & 0.88 & 64 & 0.32 & 2 & 19 & 0.02 & 4 \\
\hline P24 & 1000 & 12.1 & 296 & 0.08 & 157 & 297 & 0.31 & 274 & 0.04 & 4 & 332 & 0.00 & 14 \\
\hline P24 & 10000 & 6.0 & 94 & 0.25 & 76 & 93 & 0.52 & 133 & 0.04 & 0 & 112 & 0.00 & 6 \\
\hline P24 & 100000 & 2.7 & 12 & 0.65 & 32 & 13 & 0.85 & 56 & 0.21 & 2 & 20 & 0.02 & 3 \\
\hline P37 & 1000 & 9.8 & 434 & 0.08 & 217 & 434 & 0.30 & 379 & 0.03 & 1 & 485 & 0.00 & 19 \\
\hline P37 & 10000 & 4.9 & 150 & 0.17 & 104 & 151 & 0.46 & 181 & 0.05 & 7 & 174 & 0.00 & 9 \\
\hline P37 & 100000 & 2.6 & 23 & 0.63 & 50 & 25 & 0.82 & 87 & 0.14 & 0 & 34 & 0.00 & 5 \\
\hline P20 & 1000 & 14.9 & 263 & 0.06 & 124 & 262 & 0.25 & 217 & 0.03 & 5 & 292 & 0.00 & 10 \\
\hline P20 & 10000 & 8.9 & 62 & 0.35 & 66 & 63 & 0.66 & 116 & 0.07 & 3 & 78 & 0.00 & 6 \\
\hline P20 & 100000 & 4.4 & 4 & 0.85 & 32 & 5 & 0.94 & 55 & 0.28 & 0 & 12 & 0.06 & 3 \\
\hline P40 & 1000 & 7.1 & 481 & 0.01 & 158 & 484 & 0.10 & 275 & 0.02 & 3 & 518 & 0.00 & 15 \\
\hline P40 & 10000 & 5.1 & 155 & 0.17 & 107 & 157 & 0.44 & 187 & 0.06 & 0 & 180 & 0.00 & 10 \\
\hline P40 & 100000 & 2.9 & 32 & 0.59 & 60 & 33 & 0.80 & 106 & 0.13 & 0 & 46 & 0.00 & 6 \\
\hline P29 & 1000 & 10.8 & 345 & 0.04 & 143 & 345 & 0.22 & 250 & 0.03 & 0 & 379 & 0.00 & 13 \\
\hline P29 & 10000 & 7.2 & 106 & 0.27 & 90 & 106 & 0.55 & 158 & 0.05 & 0 & 127 & 0.00 & 8 \\
\hline P29 & 100000 & 3.0 & 22 & 0.56 & 35 & 24 & 0.79 & 61 & 0.12 & 4 & 29 & 0.00 & 4 \\
\hline P25 & 1000 & 7.8 & 308 & 0.03 & 118 & 308 & 0.16 & 206 & 0.02 & 1 & 336 & 0.00 & 10 \\
\hline P25 & 10000 & 7.8 & 78 & 0.16 & 56 & 82 & 0.50 & 98 & 0.06 & 2 & 90 & 0.00 & 6 \\
\hline P25 & 100000 & 3.8 & 21 & 0.47 & 28 & 22 & 0.71 & 49 & 0.09 & 0 & 27 & 0.00 & 3 \\
\hline P34 & 1000 & 5.9 & 404 & 0.01 & 157 & 405 & 0.17 & 273 & 0.02 & 8 & 441 & 0.00 & 14 \\
\hline P34 & 10000 & 5.9 & 119 & 0.10 & 72 & 122 & 0.39 & 126 & 0.04 & 1 & 135 & 0.00 & 7 \\
\hline P34 & 100000 & 3.8 & 30 & 0.50 & 44 & 32 & 0.74 & 77 & 0.11 & 3 & 40 & 0.00 & 4 \\
\hline P30 & 1000 & 6.8 & 372 & 0.00 & 119 & 377 & 0.08 & 207 & 0.01 & 0 & 399 & 0.00 & 11 \\
\hline P30 & 10000 & 6.5 & 89 & 0.13 & 55 & 89 & 0.40 & 96 & 0.04 & 0 & 102 & 0.00 & 5 \\
\hline P30 & 100000 & 4.3 & 27 & 0.40 & 34 & 28 & 0.69 & 59 & 0.10 & 0 & 34 & 0.00 & 3 \\
\hline
\end{tabular}


Table A3: Results for the estimators in the computer experiments. $n=10$

\begin{tabular}{|c|c|c|c|c|c|c|c|c|c|c|c|c|c|}
\hline \multirow[b]{2}{*}{ Problem } & \multirow[b]{2}{*}{ Iter. } & \multirow[b]{2}{*}{$\mathrm{SR}$} & \multicolumn{3}{|c|}{$\mathrm{JK}\left(1^{\mathrm{st}}\right)$} & \multicolumn{3}{|c|}{$\mathrm{JK}\left(2^{\mathrm{nd}}\right)$} & \multicolumn{2}{|c|}{ Weibull } & \multirow{2}{*}{$\begin{array}{c}\hat{\theta}_{W, G} \\
\text { Bias }\end{array}$} & \multicolumn{2}{|c|}{ Gumbel } \\
\hline & & & Bias & Cov. & Length & Bias & Cov. & Length & Cov. & Length & & Cov. & Length \\
\hline P11 & 1000 & 7.5 & 3 & 0.93 & 62 & 1 & 0.98 & 109 & 1.00 & 77 & 15 & 0.97 & 66 \\
\hline P11 & 10000 & 0.2 & 0 & 1.00 & 0 & 0 & 1.00 & 0 & 1.00 & 0 & 0 & 1.00 & 3 \\
\hline P1 & 1000 & 4.9 & 0 & 1.00 & 7 & 0 & 1.00 & 19 & 1.00 & 25 & 0 & 1.00 & 42 \\
\hline P1 & 10000 & 0.0 & 0 & 1.00 & 0 & 0 & 1.00 & 0 & 1.00 & 0 & 0 & 1.00 & 0 \\
\hline P16 & 1000 & 8.2 & 2 & 0.93 & 68 & 1 & 0.99 & 123 & 1.00 & 99 & 14 & 0.98 & 72 \\
\hline P16 & 10000 & 0.9 & 0 & 1.00 & 1 & 0 & 1.00 & 2 & 1.00 & 2 & 0 & 1.00 & 12 \\
\hline P6 & 1000 & 5.7 & -5 & 0.97 & 57 & -3 & 1.00 & 101 & 1.00 & 66 & 4 & 0.97 & 47 \\
\hline P6 & 10000 & 0.0 & 0 & 1.00 & 0 & 0 & 1.00 & 0 & 1.00 & 0 & 0 & 1.00 & 0 \\
\hline P26 & 1000 & 9.3 & 17 & 0.82 & 209 & 6 & 0.96 & 357 & 0.99 & 197 & 62 & 0.51 & 65 \\
\hline P26 & 10000 & 1.2 & 0 & 1.00 & 2 & 0 & 1.00 & 4 & 1.00 & 5 & 0 & 1.00 & 20 \\
\hline P21 & 1000 & 12.4 & 28 & 0.87 & 202 & 22 & 0.95 & 349 & 1.00 & 220 & 68 & 0.69 & 97 \\
\hline P38 & 1000 & 8.3 & 53 & 0.80 & 180 & 48 & 0.94 & 310 & 1.00 & 226 & 89 & 0.38 & 69 \\
\hline P21 & 10000 & 1.1 & 0 & 1.00 & 0 & 0 & 1.00 & 0 & 1.00 & 0 & 0 & 1.00 & 18 \\
\hline P38 & 10000 & 2.3 & 0 & 1.00 & 10 & 0 & 1.00 & 23 & 1.00 & 26 & 0 & 1.00 & 36 \\
\hline P31 & 1000 & 9.9 & 16 & 0.85 & 181 & 7 & 0.93 & 309 & 1.00 & 179 & 54 & 0.73 & 89 \\
\hline P31 & 10000 & 0.8 & 0 & 1.00 & 1 & 0 & 1.00 & 2 & 1.00 & 2 & 0 & 1.00 & 14 \\
\hline P35 & 1000 & 8.2 & 53 & 0.77 & 180 & 72 & 0.87 & 291 & 0.97 & 185 & 89 & 0.27 & 90 \\
\hline P35 & 10000 & 2.9 & 0 & 1.00 & 1 & 0 & 1.00 & 4 & 1.00 & 8 & 0 & 1.00 & 51 \\
\hline P7 & 1000 & 11.3 & 33 & 0.71 & 90 & 29 & 0.88 & 155 & 0.99 & 89 & 52 & 0.60 & 64 \\
\hline P7 & 10000 & 1.0 & 0 & 1.00 & 5 & 0 & 1.00 & 12 & 0.99 & 8 & 0 & 0.99 & 7 \\
\hline $\mathrm{P} 2$ & 1000 & 10.0 & 3 & 0.94 & 55 & 2 & 0.99 & 97 & 1.00 & 66 & 14 & 0.90 & 41 \\
\hline $\mathrm{P} 2$ & 10000 & 1.5 & 0 & 1.00 & 6 & 0 & 1.00 & 14 & 1.00 & 10 & 0 & 0.99 & 7 \\
\hline P3 & 1000 & 12.5 & -4 & 0.94 & 77 & -6 & 0.96 & 133 & 1.00 & 83 & 11 & 0.94 & 53 \\
\hline P3 & 10000 & 1.3 & 0 & 1.00 & 0 & 0 & 1.00 & 0 & 1.00 & 0 & 0 & 1.00 & 11 \\
\hline P27 & 1000 & 9.4 & 92 & 0.56 & 167 & 81 & 0.78 & 285 & 0.96 & 168 & 128 & 0.06 & 57 \\
\hline P27 & 10000 & 2.8 & -1 & 0.99 & 24 & -1 & 1.00 & 43 & 1.00 & 32 & 2 & 0.98 & 26 \\
\hline P17 & 1000 & 11.6 & 93 & 0.59 & 156 & 86 & 0.83 & 269 & 0.95 & 175 & 125 & 1.00 & 30 \\
\hline P17 & 10000 & 3.3 & -1 & 0.98 & 14 & -1 & 0.99 & 26 & 1.00 & 20 & 1 & 1.00 & 19 \\
\hline P22 & 1000 & 9.8 & 145 & 0.32 & 154 & 137 & 0.61 & 266 & 0.76 & 162 & 177 & 0.01 & 72 \\
\hline $\mathrm{P} 22$ & 10000 & 3.4 & -2 & 0.99 & 29 & 0 & 1.00 & 52 & 1.00 & 41 & 1 & 1.00 & 36 \\
\hline P12 & 1000 & 13.6 & 37 & 0.78 & 155 & 31 & 0.93 & 269 & 1.00 & 173 & 69 & 0.57 & 80 \\
\hline P12 & 10000 & 1.6 & 0 & 1.00 & 1 & 0 & 1.00 & 2 & 1.00 & 3 & 0 & 1.00 & 19 \\
\hline P36 & 1000 & 10.0 & 145 & 0.44 & 203 & 129 & 0.68 & 349 & 0.89 & 201 & 191 & 0.03 & 85 \\
\hline P36 & 10000 & 1.5 & 4 & 1.00 & 57 & 0 & 0.96 & 97 & 1.00 & 58 & 16 & 0.78 & 29 \\
\hline P39 & 1000 & 9.7 & 173 & 0.40 & 194 & 160 & 0.65 & 334 & 0.84 & 213 & 215 & 0.00 & 69 \\
\hline P39 & 10000 & 3.6 & 0 & 0.93 & 58 & -2 & 0.97 & 99 & 0.99 & 63 & 11 & 0.82 & 29 \\
\hline P32 & 1000 & 10.3 & 145 & 0.39 & 160 & 142 & 0.77 & 280 & 0.85 & 183 & 176 & 0.01 & 83 \\
\hline P32 & 10000 & 3.7 & -2 & 0.97 & 34 & -1 & 1.00 & 60 & 1.00 & 48 & 4 & 0.99 & 38 \\
\hline P4 & 1000 & 14.0 & 48 & 0.56 & 82 & 43 & 0.77 & 142 & 0.95 & 92 & 66 & 0.09 & 35 \\
\hline P4 & 10000 & 2.3 & 0 & 1.00 & 4 & 0 & 1.00 & 8 & 1.00 & 7 & 0 & 1.00 & 10 \\
\hline P5 & 1000 & 20.0 & 29 & 0.62 & 53 & 27 & 0.86 & 92 & 0.97 & 55 & 40 & 0.11 & 22 \\
\hline P5 & 10000 & 3.8 & 0 & 1.00 & 2 & 0 & 1.00 & 4 & 1.00 & 4 & 0 & 1.00 & 8 \\
\hline P8 & 1000 & 16.3 & 107 & 0.58 & 176 & 98 & 0.80 & 300 & 0.85 & 153 & 144 & 0.02 & 50 \\
\hline P8 & 10000 & 4.2 & -1 & 0.96 & 16 & -1 & 0.99 & 29 & 1.00 & 23 & 2 & 0.99 & 22 \\
\hline P13 & 1000 & 10.4 & 185 & 0.11 & 94 & 177 & 0.28 & 162 & 0.09 & 100 & 205 & 0.00 & 38 \\
\hline P13 & 10000 & 5.9 & 15 & 0.76 & 40 & 14 & 0.91 & 68 & 0.99 & 42 & 23 & 0.51 & 25 \\
\hline P13 & 100000 & 0.9 & 0 & 1.00 & 2 & 0 & 1.00 & 4 & 1.00 & 4 & 0 & 1.00 & 6 \\
\hline
\end{tabular}




\begin{tabular}{|c|c|c|c|c|c|c|c|c|c|c|c|c|c|}
\hline P9 & 1000 & 15.9 & 110 & 0.28 & 98 & 106 & 0.59 & 167 & 0.37 & 98 & 130 & 0.00 & 32 \\
\hline P9 & 10000 & 6.3 & 6 & 0.84 & 32 & 5 & 0.94 & 55 & 0.99 & 32 & 13 & 0.60 & 15 \\
\hline P9 & 100000 & 2.6 & -1 & 0.97 & 13 & -1 & 0.99 & 24 & 0.97 & 15 & 1 & 0.88 & 6 \\
\hline P18 & 1000 & 9.7 & 215 & 0.10 & 116 & 207 & 0.32 & 198 & 0.06 & 114 & 241 & 0.00 & 33 \\
\hline P18 & 10000 & 5.6 & 45 & 0.41 & 53 & 42 & 0.66 & 91 & 0.79 & 53 & 56 & 0.01 & 22 \\
\hline P18 & 100000 & 1.2 & 0 & 0.80 & 5 & 0 & 0.96 & 9 & 1.00 & 5 & 1 & 0.89 & 8 \\
\hline P10 & 1000 & 17.7 & 95 & 0.13 & 54 & 90 & 0.33 & 93 & 0.16 & 57 & 107 & 0.00 & 17 \\
\hline P10 & 10000 & 9.0 & 15 & 0.32 & 19 & 13 & 0.66 & 33 & 1.00 & 21 & 19 & 0.11 & 11 \\
\hline P10 & 100000 & 2.7 & 0 & 1.00 & 1 & 0 & 1.00 & 3 & 1.00 & 4 & 0 & 1.00 & 5 \\
\hline P23 & 1000 & 11.6 & 288 & 0.02 & 105 & 282 & 0.19 & 181 & 0.02 & 113 & 310 & 0.00 & 48 \\
\hline P23 & 10000 & 7.2 & 68 & 0.34 & 69 & 65 & 0.65 & 118 & 0.52 & 71 & 83 & 0.03 & 28 \\
\hline P23 & 100000 & 1.6 & -1 & 0.93 & 12 & -1 & 0.98 & 20 & 1.00 & 12 & 2 & 0.92 & 7 \\
\hline P14 & 1000 & 13.0 & 196 & 0.06 & 94 & 189 & 0.27 & 160 & 0.05 & 94 & 217 & 0.00 & 30 \\
\hline P14 & 10000 & 8.4 & 40 & 0.45 & 53 & 38 & 0.78 & 91 & 0.82 & 52 & 51 & 0.01 & 19 \\
\hline P14 & 100000 & 2.3 & 0 & 0.96 & 4 & 0 & 0.99 & 8 & 1.00 & 7 & 1 & 1.00 & 9 \\
\hline P28 & 1000 & 10.1 & 294 & 0.00 & 102 & 289 & 0.11 & 174 & 0.01 & 105 & 315 & 0.00 & 37 \\
\hline P28 & 10000 & 6.3 & 70 & 0.43 & 79 & 63 & 0.61 & 134 & 0.70 & 64 & 87 & 0.00 & 17 \\
\hline P28 & 100000 & 2.0 & 2 & 0.81 & 15 & 1 & 0.94 & 26 & 1.00 & 15 & 5 & 0.68 & 8 \\
\hline P19 & 1000 & 11.5 & 243 & 0.00 & 64 & 241 & 0.03 & 110 & 0.00 & 70 & 256 & 0.00 & 29 \\
\hline P19 & 10000 & 6.5 & 68 & 0.13 & 41 & 66 & 0.38 & 70 & 0.10 & 42 & 76 & 0.00 & 14 \\
\hline P19 & 100000 & 2.2 & 4 & 0.62 & 7 & 4 & 0.88 & 12 & 0.97 & 8 & 5 & 0.68 & 7 \\
\hline P15 & 1000 & 17.8 & 164 & 0.04 & 80 & 156 & 0.29 & 137 & 0.04 & 73 & 182 & 0.00 & 25 \\
\hline P15 & 10000 & 9.9 & 36 & 0.24 & 32 & 34 & 0.53 & 56 & 0.72 & 34 & 43 & 0.00 & 15 \\
\hline P15 & 100000 & 2.6 & 1 & 0.86 & 7 & 1 & 0.97 & 11 & 1.00 & 7 & 2 & 0.86 & 5 \\
\hline P33 & 1000 & 9.1 & 361 & 0.00 & 113 & 353 & 0.09 & 192 & 0.03 & 115 & 386 & 0.00 & 31 \\
\hline P33 & 10000 & 5.2 & 118 & 0.02 & 53 & 116 & 0.20 & 92 & 0.03 & 56 & 129 & 0.00 & 18 \\
\hline P33 & 100000 & 2.1 & 10 & 0.56 & 18 & 7 & 0.84 & 33 & 0.99 & 20 & 14 & 0.21 & 9 \\
\hline P24 & 1000 & 13.8 & 271 & 0.04 & 120 & 260 & 0.22 & 204 & 0.06 & 111 & 298 & 0.00 & 25 \\
\hline P24 & 10000 & 6.4 & 89 & 0.04 & 45 & 88 & 0.26 & 77 & 0.04 & 47 & 99 & 0.00 & 13 \\
\hline P24 & 100000 & 3.0 & 9 & 0.65 & 21 & 8 & 0.80 & 36 & 0.97 & 19 & 13 & 0.10 & 6 \\
\hline P37 & 1000 & 10.9 & 414 & 0.00 & 140 & 405 & 0.12 & 238 & 0.02 & 134 & 444 & 0.00 & 42 \\
\hline P37 & 10000 & 5.4 & 143 & 0.04 & 63 & 140 & 0.21 & 107 & 0.03 & 63 & 156 & 0.00 & 20 \\
\hline P37 & 100000 & 2.8 & 22 & 0.42 & 25 & 21 & 0.75 & 43 & 0.73 & 25 & 27 & 0.05 & 13 \\
\hline P20 & 1000 & 16.5 & 249 & 0.02 & 83 & 243 & 0.12 & 142 & 0.04 & 86 & 267 & 0.00 & 22 \\
\hline P20 & 10000 & 9.6 & 59 & 0.15 & 39 & 57 & 0.38 & 67 & 0.18 & 40 & 67 & 0.00 & 14 \\
\hline P20 & 100000 & 4.8 & 5 & 0.78 & 13 & 5 & 0.95 & 24 & 1.00 & 17 & 7 & 0.54 & 8 \\
\hline P40 & 1000 & 7.9 & 467 & 0.00 & 105 & 456 & 0.06 & 181 & 0.02 & 117 & 490 & 0.00 & 32 \\
\hline P40 & 10000 & 5.4 & 142 & 0.11 & 76 & 133 & 0.32 & 130 & 0.10 & 81 & 160 & 0.00 & 20 \\
\hline P40 & 100000 & 3.3 & 28 & 0.48 & 36 & 26 & 0.77 & 62 & 0.82 & 37 & 35 & 0.01 & 12 \\
\hline P29 & 1000 & 11.8 & 328 & 0.01 & 99 & 320 & 0.10 & 169 & 0.02 & 92 & 349 & 0.00 & 26 \\
\hline P29 & 10000 & 7.9 & 98 & 0.07 & 56 & 95 & 0.34 & 96 & 0.06 & 58 & 110 & 0.00 & 17 \\
\hline P29 & 100000 & 3.4 & 20 & 0.24 & 17 & 20 & 0.61 & 30 & 0.41 & 19 & 24 & 0.00 & 9 \\
\hline P25 & 1000 & 8.3 & 300 & 0.00 & 74 & 296 & 0.04 & 126 & 0.01 & 69 & 316 & 0.00 & 21 \\
\hline P25 & 10000 & 8.3 & 78 & 0.04 & 24 & 76 & 0.10 & 42 & 0.00 & 27 & 83 & 0.00 & 15 \\
\hline P25 & 100000 & 4.2 & 18 & 0.33 & 18 & 17 & 0.59 & 31 & 0.49 & 16 & 22 & 0.00 & 6 \\
\hline P34 & 1000 & 6.5 & 396 & 0.07 & 87 & 391 & 0.03 & 151 & 0.01 & 97 & 414 & 0.00 & 32 \\
\hline P34 & 10000 & 6.5 & 114 & 0.06 & 41 & 110 & 0.16 & 71 & 0.05 & 45 & 123 & 0.00 & 16 \\
\hline P34 & 100000 & 4.2 & 27 & 0.30 & 24 & 25 & 0.58 & 42 & 0.34 & 23 & 32 & 0.00 & 11 \\
\hline P30 & 1000 & 7.2 & 367 & 0.02 & 62 & 364 & 0.00 & 106 & 0.00 & 70 & 380 & 0.00 & 28 \\
\hline P30 & 10000 & 7.1 & 84 & 0.02 & 36 & 82 & 0.19 & 62 & 0.03 & 34 & 92 & 0.00 & 11 \\
\hline P30 & 100000 & 4.7 & 24 & 0.31 & 21 & 22 & 0.49 & 36 & 0.44 & 23 & 29 & 0.00 & 7 \\
\hline
\end{tabular}


Table A4: Results for the estimators in the computer experiments. $n=100$

\begin{tabular}{|c|c|c|c|c|c|c|c|c|c|c|c|c|c|}
\hline \multirow[b]{2}{*}{ Problem } & \multirow[b]{2}{*}{ Iter. } & \multirow[b]{2}{*}{$S R$} & \multicolumn{3}{|c|}{$\mathrm{JK}\left(1^{\mathrm{st}}\right)$} & \multicolumn{3}{|c|}{$\mathrm{JK}\left(2^{\mathrm{nd}}\right)$} & \multicolumn{2}{|c|}{ Weibull } & \multirow{2}{*}{$\begin{array}{c}\hat{\theta}_{W, G} \\
\text { Bias }\end{array}$} & \multicolumn{2}{|c|}{ Gumbel } \\
\hline & & & Bias & Cov. & Length & Bias & Cov. & Length & Cov. & Length & & Cov. & Lengtr \\
\hline P11 & 1000 & 7.9 & 0 & 0.86 & 15 & -1 & 0.96 & 26 & 1.00 & 87 & 2 & 1.00 & 166 \\
\hline P11 & 10000 & 0.4 & 0 & 1.00 & 0 & 0 & 1.00 & 0 & 1.00 & 0 & 0 & 1.00 & 13 \\
\hline P1 & 1000 & 5.0 & 0 & 1.00 & 0 & 0 & 1.00 & 0 & 1.00 & 23 & 0 & 1.00 & 96 \\
\hline P1 & 10000 & 0.0 & 0 & 1.00 & 0 & 0 & 1.00 & 0 & 1.00 & 0 & 0 & 1.00 & 0 \\
\hline P16 & 1000 & 8.4 & -1 & 1.00 & 10 & 0 & 1.00 & 19 & 1.00 & 112 & 1 & 1.00 & 186 \\
\hline P16 & 10000 & 1.0 & 0 & 1.00 & 0 & 0 & 1.00 & 0 & 1.00 & 0 & 0 & 1.00 & 28 \\
\hline P6 & 1000 & 6.0 & 0 & 1.00 & 0 & 0 & 1.00 & 1 & 1.00 & 67 & 0 & 1.00 & 132 \\
\hline P6 & 10000 & 0.0 & 0 & 1.00 & 0 & 0 & 1.00 & 0 & 1.00 & 0 & 0 & 1.00 & 0 \\
\hline P26 & 1000 & 9.6 & 4 & 0.92 & 51 & 2 & 0.99 & 89 & 1.00 & 226 & 13 & 1.00 & 191 \\
\hline P26 & 10000 & 1.5 & 0 & 1.00 & 0 & 0 & 1.00 & 0 & 1.00 & 5 & 0 & 1.00 & 54 \\
\hline P21 & 1000 & 12.7 & -1 & 0.74 & 86 & -8 & 0.79 & 147 & 1.00 & 246 & 16 & 1.00 & 261 \\
\hline P38 & 1000 & 8.4 & 33 & 0.67 & 56 & 34 & 0.93 & 98 & 1.00 & 221 & 41 & 1.00 & 194 \\
\hline P21 & 10000 & 1.6 & 0 & 1.00 & 0 & 0 & 1.00 & 0 & 1.00 & 0 & 0 & 1.00 & 53 \\
\hline P38 & 10000 & 2.4 & 0 & 1.00 & 0 & 0 & 1.00 & 0 & 1.00 & 20 & 0 & 1.00 & 86 \\
\hline P31 & 1000 & 10.2 & -3 & 0.97 & 55 & -3 & 0.99 & 96 & 1.00 & 203 & 5 & 1.00 & 229 \\
\hline P31 & 10000 & 1.1 & 0 & 1.00 & 0 & 0 & 1.00 & 0 & 1.00 & 1 & 0 & 1.00 & 40 \\
\hline P35 & 1000 & 8.4 & 33 & 0.67 & 56 & 74 & 0.37 & 70 & 1.00 & 213 & 41 & 1.00 & 239 \\
\hline P35 & 10000 & 3.3 & 0 & 1.00 & 0 & 0 & 1.00 & 0 & 1.00 & 1 & 0 & 1.00 & 121 \\
\hline P7 & 1000 & 11.6 & 21 & 0.59 & 30 & 21 & 0.81 & 52 & 1.00 & 109 & 27 & 1.00 & 165 \\
\hline P7 & 10000 & 1.0 & 0 & 1.00 & 0 & 0 & 1.00 & 0 & 1.00 & 8 & 0 & 1.00 & 17 \\
\hline P2 & 1000 & 10.5 & -1 & 0.94 & 17 & -1 & 0.97 & 30 & 1.00 & 74 & 1 & 1.00 & 108 \\
\hline P2 & 10000 & 1.5 & 0 & 1.00 & 0 & 0 & 1.00 & 0 & 1.00 & 12 & 0 & 1.00 & 18 \\
\hline P3 & 1000 & 12.8 & 0 & 1.00 & 5 & 0 & 1.00 & 10 & 1.00 & 79 & 0 & 1.00 & 144 \\
\hline P3 & 10000 & 1.9 & 0 & 1.00 & 0 & 0 & 1.00 & 0 & 1.00 & 0 & 0 & 1.00 & 30 \\
\hline P27 & 1000 & 9.7 & 68 & 0.26 & 61 & 69 & 0.68 & 108 & 1.00 & 194 & 77 & 1.00 & 158 \\
\hline P27 & 10000 & 3.0 & 0 & 1.00 & 1 & 0 & 1.00 & 3 & 1.00 & 33 & 0 & 1.00 & 71 \\
\hline P17 & 1000 & 11.8 & 83 & 0.03 & 36 & 84 & 0.16 & 63 & 1.00 & 186 & 88 & 1.00 & 79 \\
\hline P17 & 10000 & 3.6 & 0 & 1.00 & 0 & 0 & 1.00 & 0 & 1.00 & 19 & 0 & 1.00 & 54 \\
\hline P22 & 1000 & 9.9 & 106 & 0.31 & 96 & 107 & 0.68 & 162 & 1.00 & 193 & 122 & 0.99 & 174 \\
\hline P22 & 10000 & 3.7 & 0 & 1.00 & 0 & 0 & 1.00 & 0 & 1.00 & 37 & 0 & 1.00 & 93 \\
\hline P12 & 1000 & 13.9 & 32 & 0.29 & 30 & 34 & 0.71 & 56 & 1.00 & 192 & 35 & 1.00 & 216 \\
\hline P12 & 10000 & 1.8 & 0 & 1.00 & 0 & 0 & 1.00 & 0 & 1.00 & 2 & 0 & 1.00 & 43 \\
\hline P36 & 1000 & 10.5 & 92 & 0.48 & 117 & 87 & 0.77 & 196 & 1.00 & 245 & 114 & 1.00 & 204 \\
\hline P36 & 10000 & 3.3 & -1 & 0.94 & 15 & -1 & 0.98 & 27 & 1.00 & 63 & 1 & 1.00 & 75 \\
\hline P39 & 1000 & 10.0 & 123 & 0.38 & 109 & 118 & 0.55 & 184 & 1.00 & 248 & 144 & 0.88 & 174 \\
\hline P39 & 10000 & 3.7 & -1 & 0.97 & 10 & 0 & 0.98 & 18 & 1.00 & 69 & 0 & 1.00 & 85 \\
\hline P32 & 1000 & 10.6 & 129 & 0.00 & 49 & 126 & 0.18 & 85 & 1.00 & 209 & 138 & 1.00 & 228 \\
\hline P32 & 10000 & 3.8 & 0 & 1.00 & 2 & 0 & 1.00 & 5 & 1.00 & 49 & 0 & 1.00 & 100 \\
\hline P4 & 1000 & 14.4 & 38 & 0.19 & 27 & 40 & 0.50 & 48 & 1.00 & 106 & 41 & 1.00 & 91 \\
\hline P4 & 10000 & 2.4 & 0 & 1.00 & 0 & 0 & 1.00 & 0 & 1.00 & 5 & 0 & 1.00 & 24 \\
\hline P5 & 1000 & 20.2 & 26 & 0.05 & 13 & 25 & 0.35 & 23 & 1.00 & 62 & 28 & 1.00 & 61 \\
\hline P5 & 10000 & 4.0 & 0 & 1.00 & 0 & 0 & 1.00 & 0 & 1.00 & 3 & 0 & 1.00 & 18 \\
\hline P8 & 1000 & 16.7 & 100 & 0.00 & 34 & 99 & 0.12 & 59 & 1.00 & 173 & 107 & 0.98 & 154 \\
\hline P8 & 10000 & 4.3 & 0 & 1.00 & 0 & 0 & 1.00 & 1 & 1.00 & 22 & 0 & 1.00 & 57 \\
\hline P13 & 1000 & 10.7 & 158 & 0.00 & 57 & 156 & 0.15 & 97 & 0.01 & 128 & 168 & 0.00 & 88 \\
\hline
\end{tabular}




\begin{tabular}{|c|c|c|c|c|c|c|c|c|c|c|c|c|c|}
\hline P13 & 10000 & 6.2 & 15 & 0.05 & 7 & 15 & 0.23 & 12 & 1.00 & 45 & 15 & 1.00 & 69 \\
\hline P13 & 100000 & 0.9 & 0 & 1.00 & 0 & 0 & 1.00 & 0 & 1.00 & 5 & 0 & 1.00 & 13 \\
\hline P9 & 1000 & 16.3 & 99 & 0.00 & 32 & 99 & 0.13 & 55 & 0.88 & 111 & 104 & 0.20 & 92 \\
\hline P9 & 10000 & 6.5 & 6 & 0.20 & 4 & 6 & 0.59 & 8 & 1.00 & 36 & 6 & 1.00 & 41 \\
\hline P9 & 100000 & 2.6 & 0 & 1.00 & 0 & 0 & 1.00 & 0 & 1.00 & 16 & 0 & 1.00 & 18 \\
\hline P18 & 1000 & 10.0 & 191 & 0.00 & 58 & 192 & 0.05 & 98 & 0.00 & 129 & 201 & 0.00 & 88 \\
\hline P18 & 10000 & 5.6 & 36 & 0.35 & 23 & 35 & 0.37 & 40 & 1.00 & 62 & 41 & 0.99 & 57 \\
\hline P18 & 100000 & 1.3 & 0 & 0.99 & 2 & 0 & 0.99 & 3 & 1.00 & 6 & 0 & 1.00 & 19 \\
\hline P10 & 1000 & 18.4 & 74 & 0.15 & 46 & 71 & 0.39 & 78 & 0.38 & 68 & 83 & 0.00 & 39 \\
\hline P10 & 10000 & 9.4 & 4 & 0.66 & 24 & 1 & 0.66 & 42 & 1.00 & 28 & 9 & 1.00 & 24 \\
\hline P10 & 100000 & 2.7 & 0 & 1.00 & 0 & 0 & 1.00 & 0 & 1.00 & 4 & 0 & 1.00 & 11 \\
\hline P23 & 1000 & 12.0 & 268 & 0.00 & 48 & 270 & 0.00 & 88 & 0.00 & 128 & 274 & 0.00 & 123 \\
\hline P23 & 10000 & 7.6 & 61 & 0.00 & 21 & 61 & 0.08 & 36 & 0.99 & 77 & 65 & 0.77 & 76 \\
\hline P23 & 100000 & 1.7 & 0 & 1.00 & 1 & 0 & 1.00 & 2 & 1.00 & 12 & 0 & 1.00 & 20 \\
\hline P14 & 1000 & 13.4 & 181 & 0.00 & 37 & 181 & 0.00 & 64 & 0.00 & 106 & 188 & 0.00 & 79 \\
\hline P14 & 10000 & 8.6 & 36 & 0.01 & 13 & 36 & 0.09 & 22 & 1.00 & 59 & 39 & 0.97 & 54 \\
\hline P14 & 100000 & 2.5 & 0 & 1.00 & 0 & 0 & 1.00 & 1 & 1.00 & 6 & 0 & 1.00 & 24 \\
\hline P28 & 1000 & 10.4 & 289 & 0.00 & 20 & 290 & 0.00 & 36 & 0.00 & 115 & 291 & 0.00 & 106 \\
\hline P28 & 10000 & 6.5 & 50 & 0.36 & 41 & 47 & 0.53 & 70 & 1.00 & 88 & 57 & 0.05 & 42 \\
\hline P28 & 100000 & 2.0 & 1 & 0.86 & 4 & 1 & 0.96 & 7 & 1.00 & 17 & 2 & 1.00 & 21 \\
\hline P19 & 1000 & 11.7 & 236 & 0.00 & 22 & 235 & 0.00 & 38 & 0.00 & 78 & 240 & 0.00 & 81 \\
\hline P19 & 10000 & 6.7 & 60 & 0.00 & 21 & 58 & 0.38 & 35 & 0.00 & 48 & 64 & 0.00 & 38 \\
\hline P19 & 100000 & 2.3 & 4 & 0.00 & 2 & 4 & 0.16 & 3 & 1.00 & 9 & 4 & 1.00 & 19 \\
\hline P15 & 1000 & 18.3 & 155 & 0.00 & 26 & 155 & 0.00 & 45 & 0.00 & 85 & 160 & 0.00 & 66 \\
\hline P15 & 10000 & 10.2 & 25 & 0.43 & 25 & 23 & 0.63 & 43 & 1.00 & 46 & 30 & 0.78 & 34 \\
\hline P15 & 100000 & 2.8 & 1 & 0.28 & 1 & 1 & 0.66 & 2 & 1.00 & 8 & 1 & 1.00 & 12 \\
\hline P33 & 1000 & 9.4 & 332 & 0.00 & 65 & 328 & 0.00 & 111 & 0.00 & 134 & 345 & 0.00 & 78 \\
\hline P33 & 10000 & 5.3 & 115 & 0.00 & 14 & 115 & 0.00 & 24 & 0.00 & 59 & 117 & 0.00 & 53 \\
\hline P33 & 100000 & 2.1 & 4 & 0.69 & 12 & 4 & 0.80 & 17 & 1.00 & 25 & 7 & 1.00 & 22 \\
\hline P24 & 1000 & 13.9 & 239 & 0.00 & 71 & 235 & 0.11 & 120 & 0.00 & 132 & 253 & 0.00 & 64 \\
\hline P24 & 10000 & 6.6 & 84 & 0.00 & 16 & 84 & 0.01 & 27 & 0.00 & 51 & 86 & 0.00 & 38 \\
\hline P24 & 100000 & 3.1 & 7 & 0.20 & 5 & 7 & 0.64 & 10 & 1.00 & 23 & 8 & 1.00 & 17 \\
\hline P37 & 1000 & 11.2 & 396 & 0.00 & 46 & 396 & 0.00 & 81 & 0.00 & 152 & 403 & 0.00 & 116 \\
\hline P37 & 10000 & 5.5 & 133 & 0.00 & 26 & 131 & 0.00 & 45 & 0.00 & 71 & 138 & 0.00 & 55 \\
\hline P37 & 100000 & 2.9 & 20 & 0.00 & 7 & 19 & 0.08 & 12 & 1.00 & 27 & 21 & 1.00 & 35 \\
\hline P20 & 1000 & 17.2 & 225 & 0.00 & 54 & 221 & 0.00 & 92 & 0.00 & 104 & 235 & 0.00 & 53 \\
\hline P20 & 10000 & 10.0 & 48 & 0.06 & 25 & 48 & 0.29 & 42 & 0.63 & 50 & 53 & 0.00 & 34 \\
\hline P20 & 100000 & 4.8 & 4 & 0.37 & 4 & 4 & 0.70 & 7 & 1.00 & 19 & 4 & 1.00 & 20 \\
\hline P40 & 1000 & 8.1 & 428 & 0.00 & 79 & 423 & 0.00 & 133 & 0.00 & 134 & 443 & 0.00 & 68 \\
\hline P40 & 10000 & 5.7 & 118 & 0.02 & 52 & 115 & 0.36 & 89 & 0.00 & 89 & 128 & 0.00 & 43 \\
\hline P40 & 100000 & 3.4 & 24 & 0.14 & 13 & 23 & 0.36 & 22 & 1.00 & 40 & 26 & 0.98 & 37 \\
\hline P29 & 1000 & 12.2 & 298 & 0.00 & 68 & 296 & 0.00 & 115 & 0.00 & 121 & 311 & 0.00 & 62 \\
\hline P29 & 10000 & 8.1 & 96 & 0.00 & 12 & 95 & 0.00 & 21 & 0.00 & 61 & 98 & 0.00 & 51 \\
\hline P29 & 100000 & 3.4 & 19 & 0.00 & 5 & 19 & 0.01 & 8 & 0.55 & 19 & 20 & 0.97 & 25 \\
\hline P25 & 1000 & 15.3 & 281 & 0.00 & 43 & 278 & 0.00 & 74 & 0.00 & 88 & 289 & 0.00 & 54 \\
\hline P25 & 10000 & 8.6 & 72 & 0.00 & 14 & 71 & 0.00 & 24 & 0.00 & 35 & 74 & 0.00 & 37 \\
\hline P25 & 100000 & 4.3 & 15 & 0.07 & 9 & 14 & 0.43 & 15 & 0.99 & 20 & 16 & 0.26 & 15 \\
\hline P34 & 1000 & 12.8 & 373 & 0.00 & 58 & 368 & 0.00 & 99 & 0.00 & 110 & 385 & 0.00 & 82 \\
\hline P34 & 10000 & 6.7 & 98 & 0.00 & 33 & 96 & 0.14 & 56 & 0.00 & 55 & 105 & 0.00 & 36 \\
\hline P34 & 100000 & 4.3 & 26 & 0.00 & 5 & 26 & 0.01 & 10 & 0.91 & 27 & 26 & 0.70 & 28 \\
\hline P30 & 1000 & 14.5 & 361 & 0.00 & 18 & 360 & 0.00 & 31 & 0.00 & 71 & 364 & 0.00 & 75 \\
\hline P30 & 10000 & 7.4 & 80 & 0.00 & 12 & 81 & 0.00 & 20 & 0.00 & 37 & 82 & 0.00 & 29 \\
\hline P30 & 100000 & 4.8 & 19 & 0.11 & 10 & 20 & 0.28 & 18 & 0.96 & 25 & 20 & 0.08 & 17 \\
\hline
\end{tabular}




\section{$R$-code of simulated annealing}

$\mathrm{N}=100$ \# Number of candidates

$\mathrm{p}=5$ \# Number of facilities

$\mathrm{n}<-100$ \# Number of SA processes

ni<-10000 \# Number of iterations

heuristic. solution $<-\operatorname{matrix}(0$, nrow $=$ ni, ncol $=$ n $)$

heuristic.location $<-\operatorname{matrix}(0, \mathrm{nrow}=\mathrm{p}, \mathrm{ncol}=\mathrm{n})$

Store.solution<-numeric()

Best.solution<-numeric()

Store.location<-matrix $(0$, nrow $=$ ni, $n c o l=p)$

Best.location<-numeric()

for (i in 1:n)\{

subset<-numeric $(0)$

select.location<-sample $(1: \mathrm{N}, \mathrm{p}$,replace $=\mathrm{F})$

objective.function<-sum(apply(Distance.matrix[,select.location],1,min))

iteration $<-0$; Tempearture $<-400$; beta $<-0.5$ \#initial parameter setting.

while (iteration< $<$ ni) \{

sam<-sample $(1: p, 1)$

substitution<-sample $((1: \mathrm{N})[-$ select.location[sam]],1)

store.selection<-select.location

select.location[sam]<-substitution

updated.objective.function<-sum(apply(Distance.matrix[,select.location],1,min))

if (updated.objective.function $<=$ objective.function)

$\{$ objective.function<-updated.objective.function; beta<-0.5; count $<-0\}$

if (updated.objective.function>objective.function) \{

delta<-updated.objective.function-objective.function

unif.number<-runif $(1,0,1)$ 
if (unif.number<exp(-delta/Tempearture))

\{objective.function $<$-updated.objective.function; beta $<-0.5$; count $<-0$ \}

if (unif.number $>=\exp (-\mathrm{delta} /$ Tempearture $))\{$ count $<$-count +1 ; select.location $<-$

store.selection \} \}

iteration<-iteration +1

Tempearture<-Tempearture*0.95

Store.solution[iteration]<-objective.function

Best.solution[iteration]<-min(Store.solution[1:iteration])

Store.location[iteration, ]<-select.location

Best.location<-Store.location[min(which(Store.solution==Best.solution[iteration])), ]

\}

heuristic.solution[,i]<-Best.solution

heuristic.location[,i]<-Best.location

\} 